Published in: Computers and Structures, 133C, 90-102.

DOI: http://dx.doi.org/10.1016/j.compstruc.2013.11.012

\title{
Modelling and behaviour of cylindrical shell structures with helical features
}

\author{
A.J. Sadowski ${ }^{1} \&$ J.M. Rotter ${ }^{2}$
}

\begin{abstract}
Spiral welding or bonding is a particularly efficient and cost-effective method of constructing continuous tubes. However the understanding of the mechanics of such structures is not yet well developed. This is in no small part due to the difficulties involved in their computational analysis.
\end{abstract}

Cylindrical shells are traditionally modelled using rectangular finite elements oriented parallel to the meridional and circumferential axes. However, spiral features are particularly challenging to model because such features are not orthogonal to the axes of the cylinder. Commercial finite element pre-processors often struggle to mesh these with anything other than a free triangulation. A superior mesh would consist of wellconditioned rectangular elements oriented orthogonally with respect to the axes of the helix, termed a 'helical mesh', but this requires significant programming effort. A helical mesh is particularly important if features of the shell such as geometric imperfections, residual stresses, weld material and heat affected zones, and bonding in systems using adhesives are required to follow the helical form. Helically wound structural forms are widely used in different applications that demand continuous cylindrical forms. The most common uses in structural engineering are in spiral wound tubes, piles, chimneys and pipelines.

This paper describes a powerful computational procedure developed by the authors to generate high quality helical meshes. Special emphasis is placed on the modelling of geometric deviations defined relative to the helix, appropriate for the analysis of spiral welded and stiffened tubes. The effect of helical meshing is illustrated using benchmark examples of perfect and imperfect cylinders under axial compression. 
Published in: Computers and Structures, 133C, 90-102.

DOI: http://dx.doi.org/10.1016/j.compstruc.2013.11.012

\section{Keywords}

Helical meshing, spiral imperfections, residual stresses, nonlinear finite element analysis, shell buckling, imperfection sensitivity.

${ }^{1}$ Lecturer, Imperial College London, UK

${ }^{2}$ Professor of Civil Engineering, The University of Edinburgh, UK 
Published in: Computers and Structures, 133C, 90-102.

DOI: http://dx.doi.org/10.1016/j.compstruc.2013.11.012

\section{Introduction}

The most common structural application of a cylindrical shell with helical features is the spiral formed tube, first used at the end of the $19^{\text {th }}$ century in water transmission pipelines $[1,2]$. Spiral formed pipes were initially constructed by riveting together appropriately bent plates [3] until advances in welding technology allowed for efficient tandem arc welding [1] (Fig. 1). Spiral welded tubes are now widely used in applications such as water, gas and oil pipelines under both low and high pressure [4] as well as for foundation piles and primary load-bearing members in Combiwalls [5].

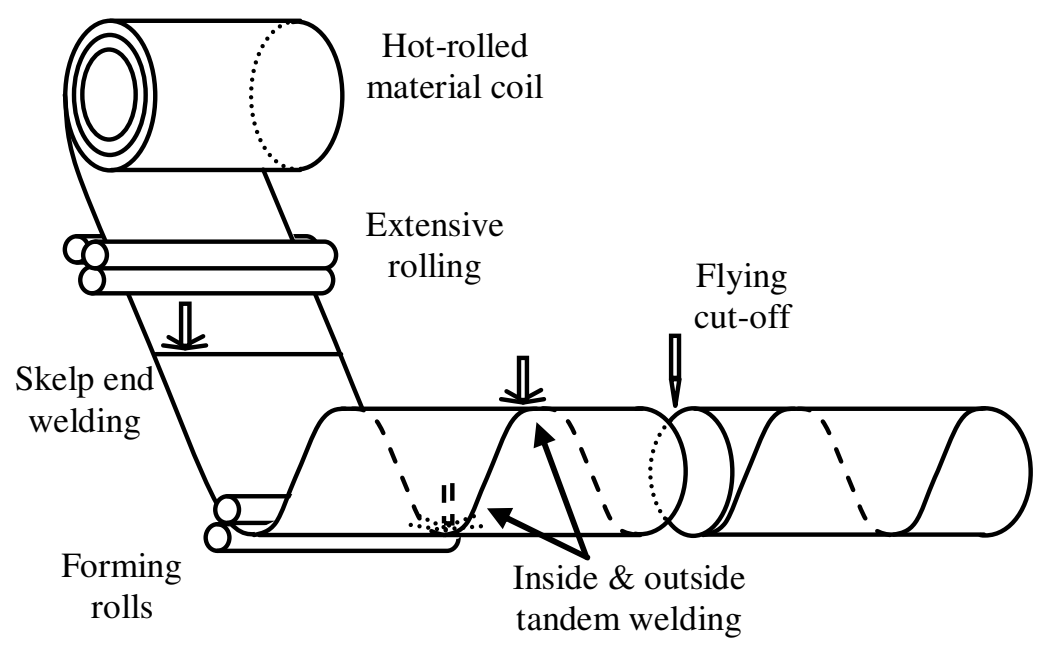

Fig. 1 - Generic illustration of key features of the spiral welding process

Spiral welded tubes offer certain advantages over traditional longitudinal and buttwelded tubes. In particular, continuous or very long tubular members may be constructed efficiently both in a factory and on-site from compact coils of metal strip, eliminating the need for costly transport of long structural members. The material coil is usually produced to very tight tolerances which results in a consistent wall thickness in the finished tube [6]. Further, under certain conditions spiral welded tubes have been found to exhibit a superior performance in fatigue tests to that of tubes with longitudinal seams [7]. They also exhibit a comparable resistance to ductile crack propagation [8]. However, the diameter and wall thickness of spiral welded tubes are currently limited to approximately $3 \mathrm{~m}$ and $30 \mathrm{~mm}$ respectively [9] which generally makes them unsuitable for offshore and deep-water applications [10].

Not every helical feature produces a detrimental imperfection. For example, tall slender chimneys are often fitted with continuous spiral fins, known as 'helical 
Published in: Computers and Structures, 133C, 90-102.

DOI: http://dx.doi.org/10.1016/j.compstruc.2013.11.012

spoilers', to protect against alternating vortex shedding that can cause large oscillations under wind [11]. Other structures include screw conveyor shafts [12], pre-stressed concrete and plastic pipes with helical wire reinforcement $[13,14]$ and specialist thinwalled cylindrical tanks constructed using either the LIPP® Dual-Seam or similar spiral folding systems.

\section{Current computer modelling of cylinders with helical features}

Spiral welded and helically wound cylindrical structures represent a fast growth area in several industries due to the above advantages. However, very little scientific study has so far been undertaken to gain an understanding of the nonlinear behaviour and mechanics of such structures, so the field is still in its infancy. The limited literature that does exist is based largely on empirical studies rather than analytical ones. This is in no small part due to the mathematical complexity of helical geometries which precluded their consideration in the development of thin-walled shell theory when the first hand solutions were being developed [15,16,17,18,19].

These difficulties have persisted into the computer era. Helical features in cylindrical structures represent a significant challenge for finite element modelling because they are not orthogonal to the principal axes of the structure and are consequently difficult to mesh, though several attempts have been made in the past decade. For example, Dong et al. [20] analysed a cylindrical spiral welded steel tube with ABAQUS [21] using a 'double-helix' mesh of inclined quadrilateral shell elements (Fig. 2). The welding process was modelled using a thermo-mechanical material model applied directly to the elements defined as the 'weld', and the tube was given no geometric deviations. Wirth [22] used ANSYS [23] to perform nonlinear buckling analyses of axially-compressed thin-walled cylinders with explicitly-modelled spiral LIPP® folds using a 'single-helix' mesh (Fig. 2) of slightly distorted shell elements. As the helical incline angle was very shallow, the mesh distortion was minimal and the analyses were in close agreement with experiments. Arif et al. [24] used ANSYS [23] to perform a thermal stress analysis of a laser-welded spiral tube with solid continuum elements. They employed a user subroutine to generate a 'single-helix' partition of the tube to trace the outline of the spiral weld for the purposes of defining an appropriate region for load introduction and material properties. However the perfect geometry of the 
Published in: Computers and Structures, 133C, 90-102.

DOI: http://dx.doi.org/10.1016/j.compstruc.2013.11.012

structure itself was meshed freely with tetrahedral elements. Ohnishi et al. [25] analysed spiral fabricated steel pipes under cyclic loading using a 'double-helix' mesh of shell finite elements and the CYNAS [26,27] finite element program, though the choice of mesh and its consequences were not discussed. In their study of predicted residual stress patterns in arc-welded spiral pipes, Forouzan et al. [28] used ANSYS [23] to model the spiral pipe with a 'single-helix' mesh of distorted shell elements. Rather than employing a partitioning procedure, the authors used external scripts to generate nodal coordinates of the perfect shell directly. No mention is made of the effect of element distortion on the accuracy of the solution, though the authors reported a good agreement with experiment.

Sadowski and Rotter [29] described a detailed procedure according to which a commercial finite element pre-processor may be used to model a cylindrical shell with a regular mesh of well-defined, helical, quadrilateral shell elements. As always, however, the user must be sufficiently proficient as a programmer to manually create helical partitions on the cylinder. These in turn force the intrinsic meshing algorithm of the pre-processor to generate quadrilateral elements which conform to the axis of the helix rather than to that of the cylinder. The procedure is general enough to be applied using any finite element pre-processor with an external scripting functionality (e.g. ABAQUS, ANSYS, COMSOL). This manner of 'helical meshing' is central to the material presented in this paper, so a brief outline is appropriate here.

The procedure of Sadowski and Rotter [29] proposes two partition configurations (Fig. 2), the 'single-helix' and 'double-helix', each leading to a distinctly different type of inclined finite element mesh. In the first type (Fig. 2a), a family of equally-spaced adjacent helices is generated on the cylinder surface, each helix forming an angle of $\alpha$ with the transverse axis and partitioning the cylinder face accordingly. The preprocessor then generates a regular mesh of curved parallelogram elements where one pair of opposing sides lie parallel to the horizontal axis and the other pair is parallel to the helices. Though relatively simple to code, elements generated in this manner become increasingly distorted with the helical angle $\alpha$ which may result in inferior performance [30,31]. The second, superior, type of partitioning involves generating regularly-spaced pairs of orthogonal helices. The pre-processor then creates a regular mesh of well-conditioned curved rectangular elements that all share the same angle of 
Published in: Computers and Structures, 133C, 90-102.

DOI: http://dx.doi.org/10.1016/j.compstruc.2013.11.012

inclination everywhere (Fig. 2b), except near the ends of the cylinder where some local triangulation is necessary to enforce a circular diametral boundary.

\section{a) 'Single-helix' mesh}

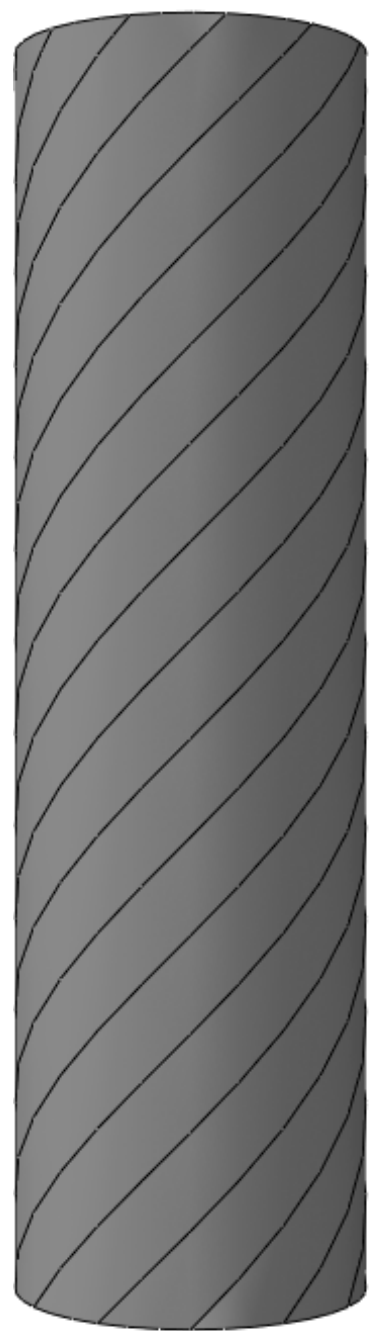

Single-helix partition

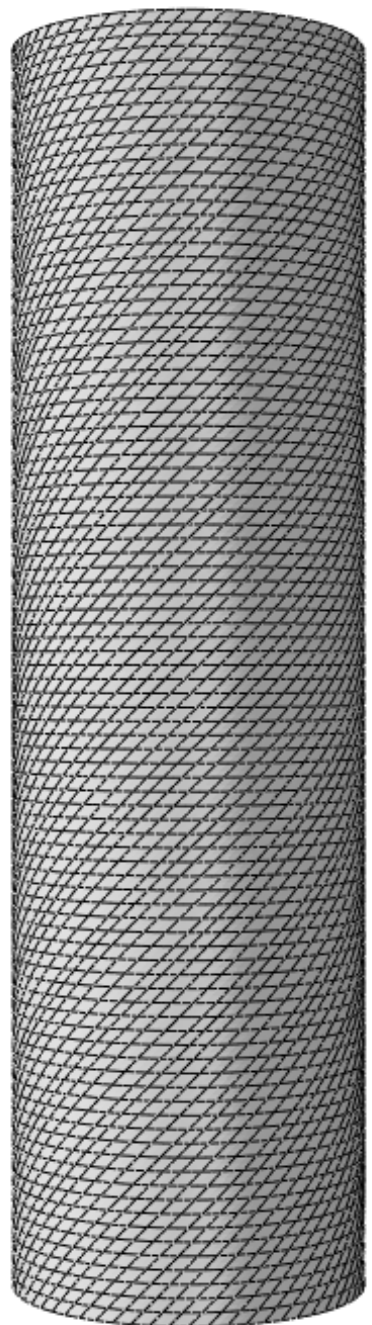

Distorted mesh

b) 'Double-helix' mesh

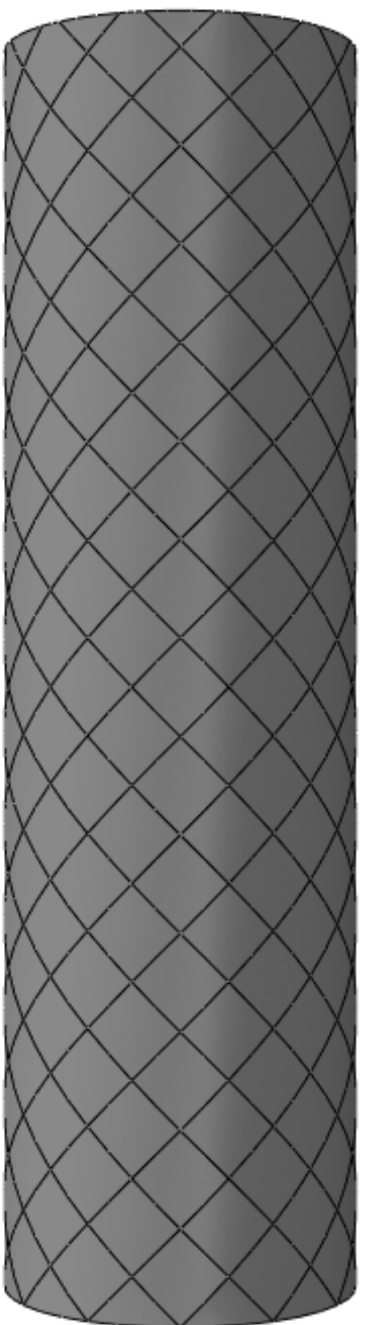

Double-helix partition

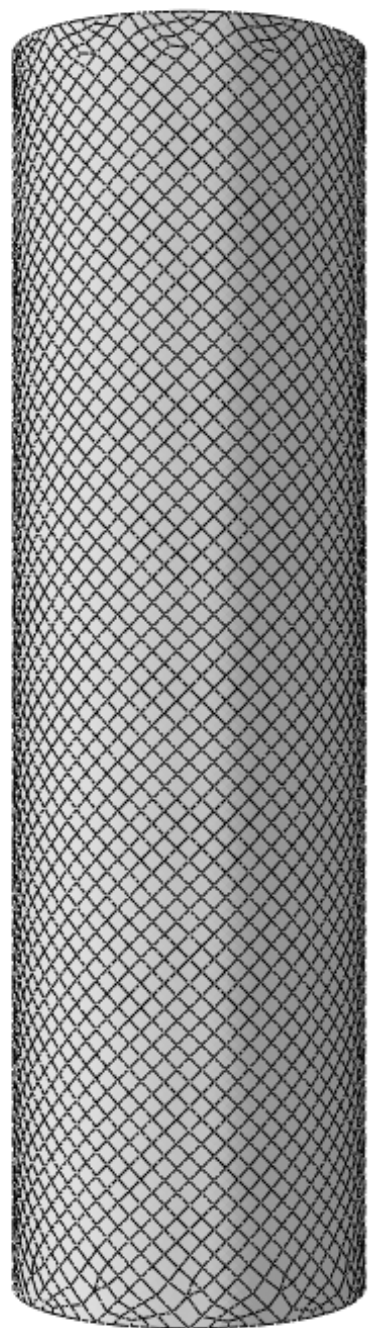

Inclined regular mesh

Fig. 2 - Illustration of helical partitioning and meshing in ABAQUS CAE, after [29]

With appropriate scripting, helical partitioning clearly works very well to model perfect cylindrical geometries. Indeed, all the computational studies referenced above used partitioning on a perfect shell to model the effects of spiral welds. However, the meshing algorithm of a typical commercial pre-processor usually struggles to mesh helically partitioned cylinders with all but the most trivial spiral geometric imperfections using any method other than a free triangulation. The more geometrically versatile triangular elements can certainly be used to mesh deep spiral 
Published in: Computers and Structures, 133C, 90-102.

DOI: http://dx.doi.org/10.1016/j.compstruc.2013.11.012

imperfections, but well-conditioned quadrilateral elements are more desirable for numerical accuracy, especially when performing sophisticated nonlinear buckling or plastic collapse analyses. It is very difficult to model cylindrical shells with significant geometric spiral imperfections in a satisfactory manner using the above methods.

\section{Parametric meshing of thin cylindrical shells with helical features}

\subsection{Introduction}

This paper illustrates a computational method to model thin cylindrical shells with helical features without the direct aid of a commercial finite element pre-processor. The method involves the generation of the entire finite element mesh in an external programming environment. The helically-wound cylinder is first conceptually 'unwound' into its constituent rectangular strip, which is then meshed with wellstructured regular rectangular elements. The nodal coordinates are then mapped back onto the cylinder using an appropriate transformation, a method known as 'parametric surface meshing' [32]. Two distinct transformations are presented here for completeness; a non-conformal transformation leading to a 'single-helix' type mesh (Fig. 2a) and a conformal transformation leading to a 'double-helix' type mesh (Fig. 2b). More details are presented below.

The freedom and versatility gained in having complete control over mesh generation outweigh the time investment necessary to write the software. In particular, the advantages of a regular and well-conditioned mesh of quadrilateral shell elements that are orthogonal to the helix include the following:

- An accurate and elegant representation of the surface geometry of the helical features.

- Convenient specification of material properties and mechanical and thermal load cases of any complexity relative to the axes of either the helix or the cylinder.

- Freedom to introduce geometric imperfections of any shape or amplitude defined relative to either the helical or cylindrical axes.

- Ability to model additional features such as spiral reinforcement, spiral stiffeners, spiral seams, spiral heat-affected zones from weld material and spiral residual stress distributions (e.g. [28,33]). 
Published in: Computers and Structures, 133C, 90-102.

DOI: http://dx.doi.org/10.1016/j.compstruc.2013.11.012

- Potential to extend the treatment to 3D solid continuum elements without any additional mathematical complexity.

It is important to stress that this meshing method is intended solely for the modelling of imperfect cylindrical shells with significant geometric deviations that are helical in nature, and not as an alternative to the traditional modelling of perfect cylindrical shells with regular rectangular meshes. The detrimental effects of the inappropriate application of helical meshing to model a perfect geometry are illustrated in detail in the next section of this paper through the classical example of the highly imperfectionsensitive perfect cylinder under uniform axial compression. This is followed by an illustration of the intended use of this meshing method, namely the analysis of a spiral welded (imperfect) cylinder under uniform axial compression.

A helix of radius $R$ and length $H$ parallel to its central axis may be defined either by the number of turns $n_{p}$ (not necessarily an integer) or by the angle of inclination $\alpha$, defined here for convenience as being relative to the circumferential axis. The 'pitch' of the helix $P$ is the change in the length of the cylinder for each complete turn, measured parallel to the central axis. The above parameters are then related by:

$$
P=2 \pi R \tan \alpha=\frac{H}{n_{p}}
$$

A right-handed helix may be defined parametrically in 3D Cartesian coordinates by:

$$
x(\theta)=R \cos \theta \quad y(\theta)=R \sin \theta \quad z(\theta)=\frac{P}{2 \pi} \theta
$$


Published in: Computers and Structures, 133C, 90-102.

DOI: http://dx.doi.org/10.1016/j.compstruc.2013.11.012

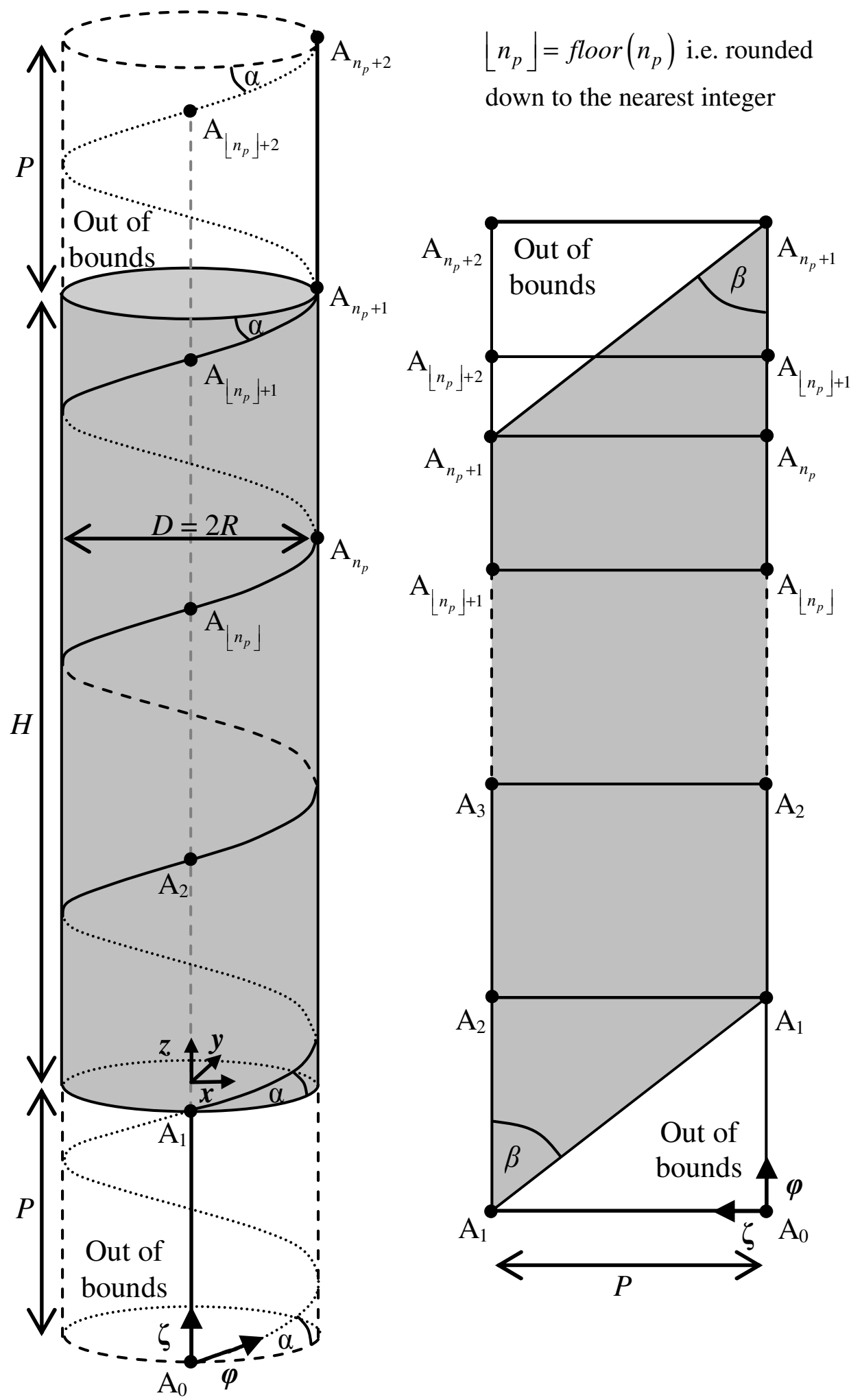

Fig. 3 - System geometry for a non-conformal transformation for a 'single-helix' mesh 
Published in: Computers and Structures, 133C, 90-102.

DOI: http://dx.doi.org/10.1016/j.compstruc.2013.11.012
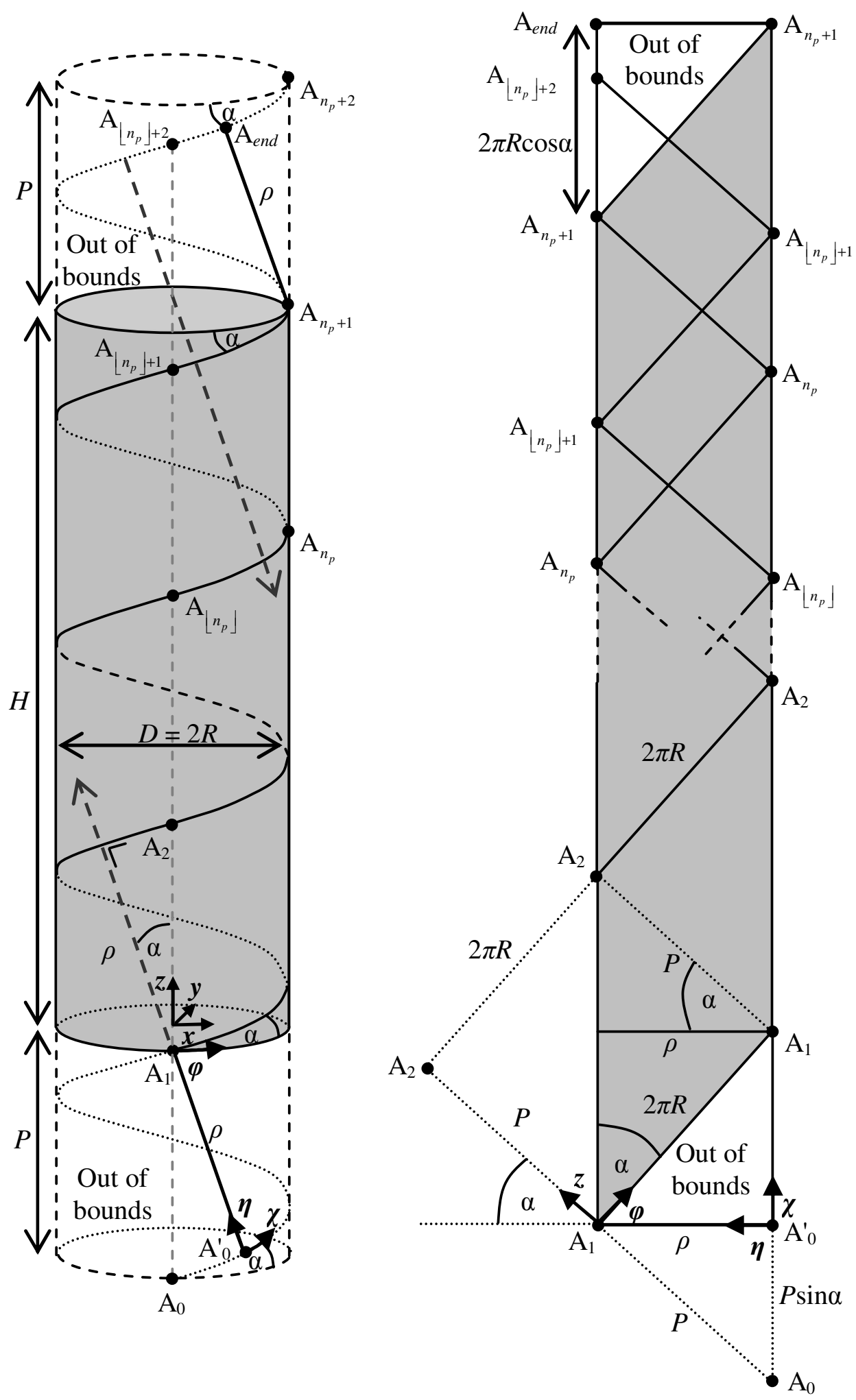

Fig. 4 - System geometry for a conformal transformation for a 'double-helix' mesh 
Published in: Computers and Structures, 133C, 90-102.

DOI: http://dx.doi.org/10.1016/j.compstruc.2013.11.012

\subsection{Regular rectangular meshing}

The Cartesian coordinates of a perfect or imperfect cylindrical shell may be generated parametrically using the following familiar formulae:

$x(\theta, z)=r(\theta, z) \cos \theta$

$y(\theta, z)=r(\theta, z) \sin \theta$

$z(\theta, z)=z$

The coordinate ranges are $0 \leq \theta \leq 2 \pi$ and $0 \leq z \leq H$, where $H$ is the length of the cylinder. Here $r(\theta, z)$ is the radial coordinate of the middle surface of the cylindrical shell which may be programmed to define a geometric imperfection. For a perfect shell, $r(\theta, z)=R$ where $R$ is the radius of the cylinder. Where the nodal coordinates of a mesh are generated according to these equations to give rectangular elements that are orthogonal to the axes of the cylinder, the result is referred to here as a 'rectangular' mesh.

\section{3 'Single-helix' helical mesh}

The nodal transformation leading to a 'single-helix' type mesh of distorted parallelogram shell elements (Figs 2a and 3) is defined as:

$$
\begin{aligned}
& x(\varphi, \zeta)=r(\varphi, \zeta) \cos [\theta(\varphi, \zeta)] \\
& y(\varphi, \zeta)=r(\varphi, \zeta) \sin [\theta(\varphi, \zeta)] \\
& z(\varphi, \zeta)=P\left(\frac{\theta(\varphi, \zeta)}{2 \pi}-1\right)+\zeta
\end{aligned}
$$

where $\theta(\varphi, \zeta)=\frac{2 \pi \varphi}{\sqrt{P^{2}+(2 \pi R)^{2}}}$

The coordinate ranges are $0 \leq \varphi \leq\left(n_{p}+1\right) \sqrt{P^{2}+(2 \pi R)^{2}}$ and $0 \leq \zeta \leq P$, where $R$ is the nominal radius of the cylinder and $P$ is the pitch of the helix (Eq. 1). The locallydefined radial coordinate of the shell wall $r(\varphi, \zeta)$ allows the specification of geometric imperfections that are rectilinear in the $\varphi-\zeta$ plane but helical in the $x-y-z$ space. For a perfect shell, $r(\varphi, \zeta)=R$. This transformation is non-conformal because it does not preserve angles. Lines with $\varphi=$ constant map to vertical lines in the $x-y-z$ space, while lines with $\zeta=$ constant (orthogonal to $\varphi=$ constant lines) map to helices in the $x-y-z$ 
Published in: Computers and Structures, 133C, 90-102.

DOI: http://dx.doi.org/10.1016/j.compstruc.2013.11.012

space which are not orthogonal to the vertical axis. The relation between the angles $\alpha$ and $\beta$ in Fig. 3 is:

$\sin \alpha=\tan \beta$

\section{4 'Double-helix' helical mesh}

The nodal transformation leading to a 'double-helix' type mesh of inclined regular rectangular shell elements (Figs $2 \mathrm{~b}$ and 4 ) is defined as:

$$
\begin{aligned}
& x(\chi, \eta)=r(\chi, \eta) \cos [\theta(\chi, \eta)] \\
& y(\chi, \eta)=r(\chi, \eta) \sin [\theta(\chi, \eta)] \\
& z(\chi, \eta)=\eta \sec \alpha+\theta(\chi, \eta) R \tan \alpha-\rho \cos \alpha
\end{aligned}
$$

where $\theta(\chi, \eta)=\frac{\cos \alpha}{R}(\chi-\eta \tan \alpha)$

The coordinate ranges are $0 \leq \chi \leq\left[2 \pi R \cos \alpha+n_{p} \sqrt{P^{2}+(2 \pi R)^{2}}\right]$ and $0 \leq \eta \leq \rho$. The locally-defined radial coordinate of the shell wall $r(\chi, \eta)$ again allows the specification of geometric imperfections that are rectilinear in the $\chi-\eta$ plane but helical in the $x-y-z$ space. For a perfect shell, $r(\chi, \eta)=R$. The parameter $\rho$ represents the 'width' of the strip in the $\chi-\eta$ plane and is given by:

$$
\rho=\frac{2 \pi R P}{\sqrt{P^{2}+(2 \pi R)^{2}}}
$$

This transformation is conformal because it preserves angles. In particular, lines with $\chi$ and $\eta=$ constant map to orthogonal helices in the $x-y-z$ space resulting in a superior three-dimensional mesh of inclined doubly-curved quadrilateral shell elements. 
Published in: Computers and Structures, 133C, 90-102.

DOI: http://dx.doi.org/10.1016/j.compstruc.2013.11.012

\subsection{Outline of computational procedure}

The following is a skeleton outline of the computational procedure used by the authors to implement the above transformations and to generate inclined helical meshes for thin-walled cylindrical structures. The output from this procedure was designed to be in the form of self-contained inp input files for direct use by the ABAQUS/Standard $\mathrm{v}$ 6.10.1 [21] finite element solver without any further processing. The coding was done within the Matlab R14 programming environment [34] enhanced with an open-source MPI parallelisation plug-in MatlabMPI developed at the MIT Lincoln Laboratory [35]. In what follows, the original $x-y-z$ space is referred to as the 'model' space while the $\varphi-\zeta$ or $\chi-\eta$ planes are referred to as the 'image' planes.

The general procedure is as follows:

- Process inputs and initialise the geometry of the model plane.

- Choose transformation type and calculate the coordinate ranges of the rectangular strip in the corresponding image plane (Figs 3 and 4).

- Mesh the image plane using regular rectangular elements of any order to cover the full coordinate ranges (Fig. 5a). The highly organised structure of this mesh is particularly advantageous for node and element numbering, and the meshing may be parallelised quite easily for great increases in processing speed.

- The circular transverse boundaries of the cylinder correspond to inclined boundaries on the strip. Thus any elements that fall on or beyond the inclined boundary of shaded regions (within tolerances), marked as 'out of bounds' on Figs 3 and 4, should be discarded along with any unnecessary related nodes (Fig. 5b). The elements that fall fully within the shaded region should be retained.

- Seed the inclined boundary of the image plane with nodes and fill the gap (dashed region, Fig. 5b) with triangular elements using any appropriate Delaunay triangulation algorithm (e.g. [32,36]). This triangulation connects the helical mesh with the circular transverse end boundaries of the cylinder (Figs $5 \mathrm{c}$ and 6$)$.

- There are two inclined boundaries, one at each end of the strip corresponding to the boundaries transverse to the cylinder at $z=0$ and $H$. They may be treated independently and this stage of the generation may thus be parallelised. It is 
Published in: Computers and Structures, 133C, 90-102.

DOI: http://dx.doi.org/10.1016/j.compstruc.2013.11.012

preferable if the order of the elements used in the triangulation is consistent with that of the rectangular elements used elsewhere.

- Since the transformations effectively cause the strip to be 'wound' round the cylinder, the line of nodes $\left[A_{1}, \ldots, A_{n p+1}\right]$ on the LHS of the strip $(\zeta=P$ or $\eta=\rho$ ) must map to the RHS of the strip $(\zeta=0$ or $\eta=0)$ to ensure nodal continuity.

- This 'mirror' boundary condition is easily achieved for a 'single-helix' mesh (Fig. 6a) because corresponding $A_{n}-A_{n+1}$ pairs on $\zeta=0$ and $P$ share the same $\eta$ coordinate. Enforcing this BC is therefore simply a case of renumbering all the nodes for $\zeta=0$ within the shaded region so that they are the same (and in the same order) as those for $\zeta=P$.

- The conformal transformation of the 'double-helix' mesh leads to a misalignment of nodal coordinates upon 'winding' (Fig. 6b) for all helical angles except $45^{\circ}$. The coordinates of the nodes within the shaded region on the line $\eta=0$ must therefore be adjusted, in addition to being renumbered, to maintain the same nodal distances as the nodes on the line $\eta=\rho$, as illustrated in Fig. 5d. Unfortunately, the rectangular elements with a side on $\eta=0$ are consequently distorted into parallelograms and may usefully be degenerated into two triangular elements of the same order. In all of the analyses presented here, care was taken to ensure that this strip of triangular elements was not used to model areas of the structure where, for example, a helical imperfection or other geometric feature has been defined, as triangular elements rarely hold any advantage over high quality rectangular elements in structural analyses.

- Arrange the output to be in a format that may be easily imported into a thirdparty package for further processing or used directly by a preferred finite element solver. 


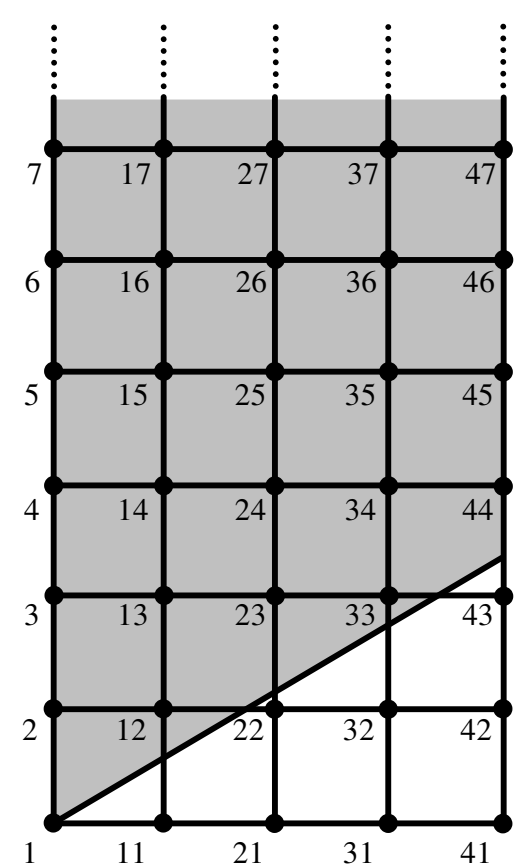

a) Rectangular meshing of strip \& ordered node / element numbering

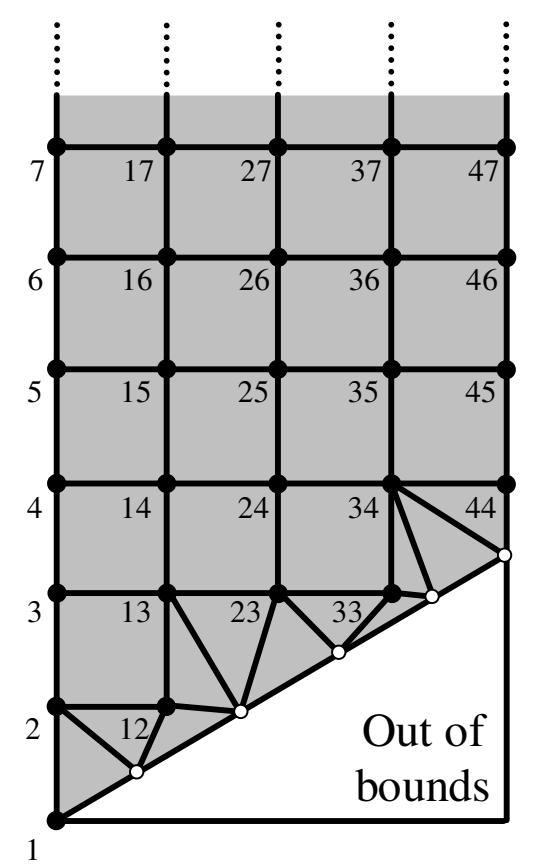

c) Node seeding of inclined boundary and triangulation

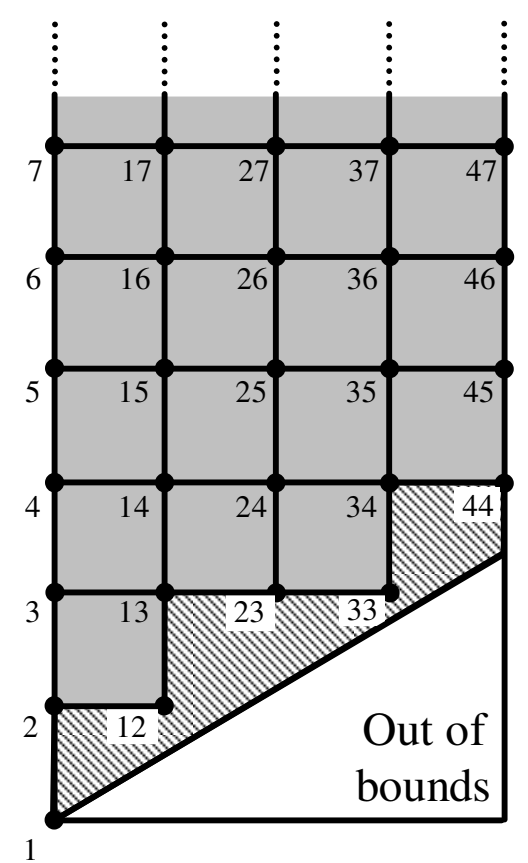

b) Elimination of all 'out of bounds' and boundary elements

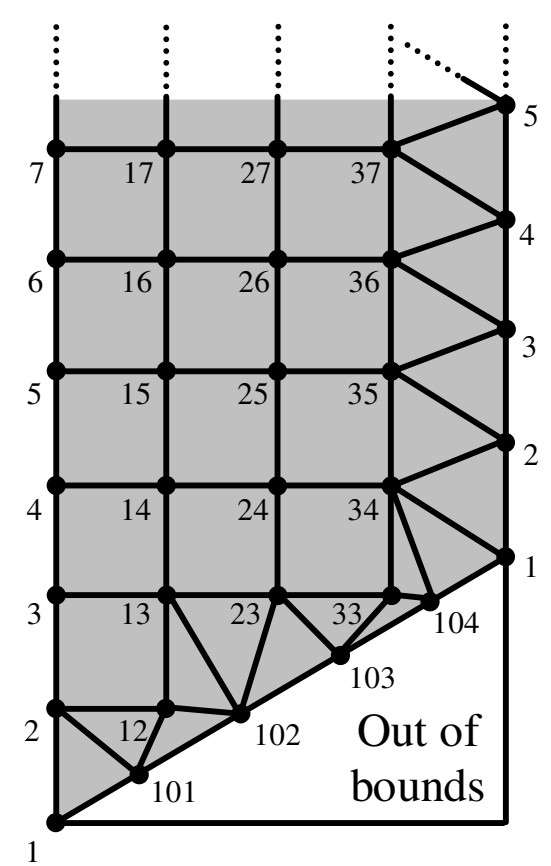

d) Node rearrangement and renumbering to enforce a mirror BC

Fig. 5 - Meshing of the rectangular strip in the image plane 


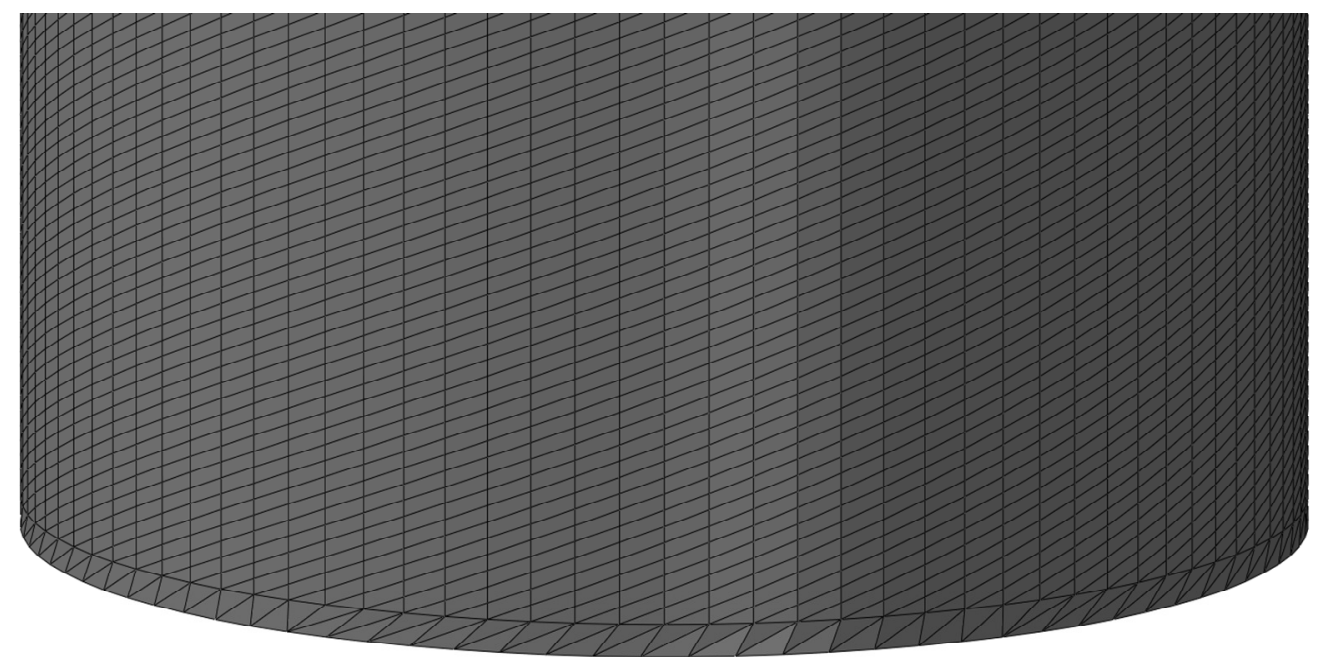

a) 'Single-helix' mesh

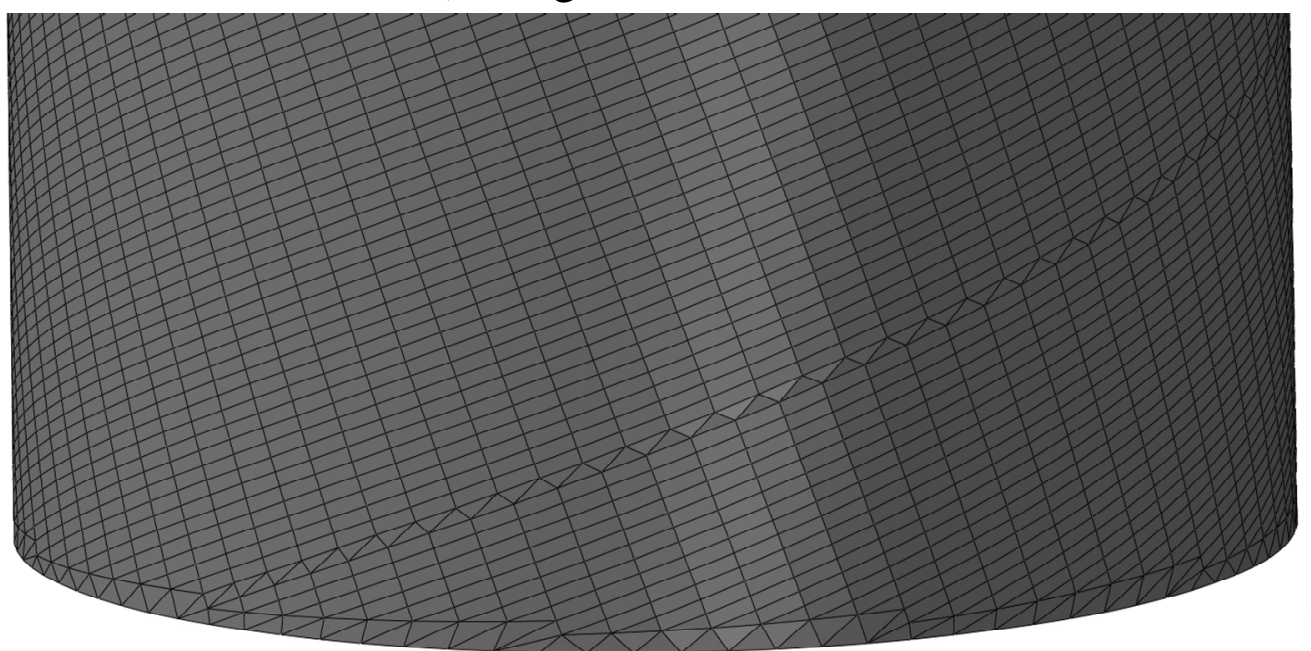

b) 'Double-helix' mesh

Fig. 6 - Cylindrical boundary details for the two helical mesh types

(helical angle $\alpha=20^{\circ}$ )

\subsection{Generation of geometric imperfections}

One of the key advantages of generating the finite element mesh in custom-written software is the ability to retain full control over the nodal coordinates. The transformations presented in this paper have been set up to facilitate the convenient definition of geometric imperfections either before or after the nodal coordinate transformation. This permits a cylinder endowed with imperfections defined in any direction to be modelled with a high-quality mesh of quadrilateral finite elements oriented optimally with respect to the imperfection. A number of potentially useful applications are presented in the remainder of this paper. 
Published in: Computers and Structures, 133C, 90-102.

DOI: http://dx.doi.org/10.1016/j.compstruc.2013.11.012

To illustrate this through a visual example, a common imperfection in cylindrical shells has traditionally been the eigenmode $[37,38,39,40,41,42]$ where the wall is deformed into sinusoidal waves in terms of the meridional and circumferential nodal coordinates. A cylinder with an eigenmode imperfection with $n_{\theta}$ circumferential and $n_{z}$ axial waves respectively may be defined in the model $x-y-z$ plane using Eqs 3 to 5 assuming:

$$
r(\theta, z)=R-\delta \sin \left(n_{\theta} \cdot \theta\right) \sin \left(n_{z} \cdot \frac{2 \pi z}{H}\right)
$$

Where the coordinates of the cylinder are generated using the helical transformations presented earlier (Eqs. 6 to 9 and 11 to 14 respectively), an eigenmode imperfection may be defined in the $x-y-z$ space as follows:

'Single-helix' mesh: $r(\varphi, \zeta)=R-\delta \sin \left[n_{\theta} \cdot \theta(\varphi, \zeta)\right] \sin \left[n_{z} \cdot \frac{2 \pi}{H} z(\varphi, \zeta)\right]$

'Double-helix' mesh: $r(\chi, \eta)=R-\delta \sin \left[n_{\theta} \cdot \theta(\chi, \eta)\right] \sin \left[n_{z} \cdot \frac{2 \pi}{H} z(\chi, \eta)\right]$

where $z(\varphi, \zeta), \theta(\varphi, \zeta), z(\chi, \eta)$ and $\theta(\chi, \eta)$ are given by Eqs. 8, 9, 13 and 14 respectively. The resulting finite element meshes (Fig. 7) illustrate how the physical geometry and imperfections can be kept exactly 'constant' while the mesh is 'rotated'.

This simple example illustrates an important application of helical meshing in the validation modelling of spiral welded cylindrical tubes with measured full 2D initial imperfection surveys. Such surveys are traditionally subject to a $2 \mathrm{D}$ Fourier analysis which disassembles the imperfections into their constituent harmonics expressed as a double trigonometric series defined in the cylindrical coordinate system, a technique which has been employed to characterise measured imperfections in both aerospace and civil engineering shells for almost half a century (e.g. [68,69,70,71,72]). The helical meshing procedure presented in this paper may readily be used to create very accurate models of spiral welded cylinders with realistic measured imperfections. 


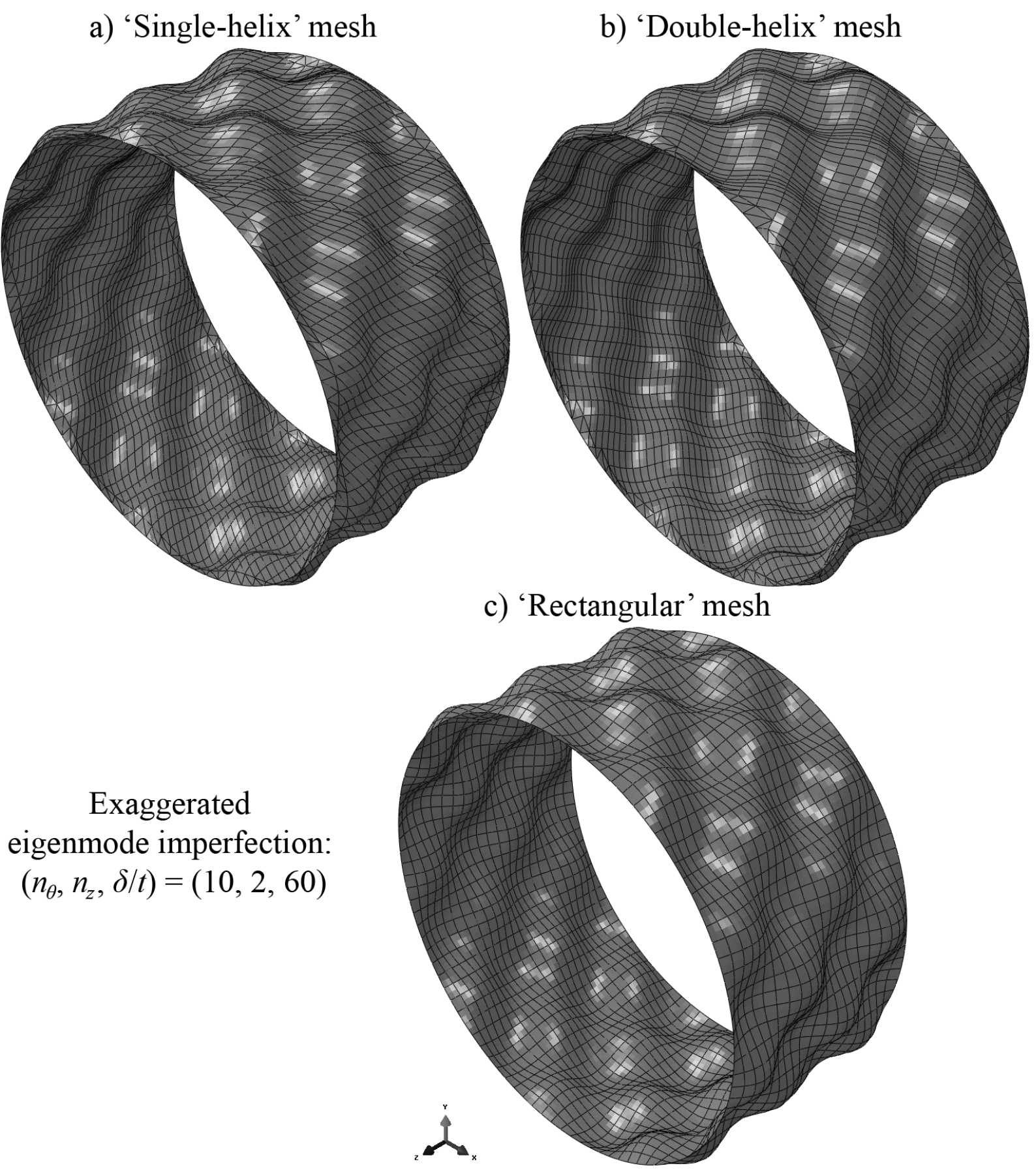

Fig. 7 - Modelling of eigenmode imperfections with rectangular and helical meshes (helical angle $\alpha=30^{\circ}$ )

\section{Verification of helical meshing - cylinder under axial compression}

The helical meshing procedure is illustrated in what follows with the help of the classical example of the buckling of perfect thin-walled cylinders under uniform axial compression. This is a ubiquitous benchmark in the field of shell buckling and has been studied very extensively in the past (e.g. $[16,19,37,40,44,45,46,47,49,52]$ ). 
Published in: Computers and Structures, 133C, 90-102.

DOI: http://dx.doi.org/10.1016/j.compstruc.2013.11.012

However, this deceptively simple problem is widely recognised to be one of the most challenging to model computationally. This may be attributed to the fact that under a state of uniform compressive stress, the critical buckling mode of the cylinder is sensitive to the slightest disturbance of any kind, physical or numerical, making this one of the most imperfection sensitive structural forms. Indeed, it will be shown that helical meshing is not an appropriate formulation for such systems and should not be employed for the modelling of nominally perfect cylindrical structures.

The analyses were performed using the commercial ABAQUS v. 6.10.1 [21] finite element software on meshes and input inp files generated externally by the authors using the methods described above. The cylinder was assumed to have a radius to thickness ratio $R / t$ of 1000 and a Batdorf dimensionless length parameter of $Z=H^{2}(1-$ $\left.v^{2}\right)^{1 / 2} /(R t)=1000$ [40] or, in terms of the alternative dimensionless length measure, $\omega=$ $H /(R t)^{1 / 2}=32.82$ [43], giving a length to diameter $H / D$ ratio of 0.512 . A length of $\omega=$ 32.82 places the shell in the 'medium' length category $[40,43,46]$, long enough so that the buckling mode is not strongly influenced by the boundary conditions but short enough to prevent Euler column buckling. The material was assumed to be isotropic mild steel with a modulus of elasticity of $E=200 \mathrm{GPa}$ and Poisson's ratio of $v=0.3$. The elements were specified to be four-node reduced-integration bilinear general purpose shell elements ( $\mathrm{S} 4 \mathrm{R}$, considered to be the most versatile of the ABAQUS library [50]) with maximum element length no longer than $0.5(R t)^{1 / 2}$. It was necessary to model the full $360^{\circ}$ of the shell circumference because it is not possible to exploit symmetry when using helical meshing. The shell was loaded with the classical elastic critical buckling stress resultant $N_{c l}$ (Eq. 19) along one edge where the radial displacement and meridional rotation (but not the meridional displacement) were restrained (clamped C3 condition [40]) while the other edge was restrained against both radial and meridional displacements and meridional rotation (clamped $\mathrm{C} 1$ condition).

$$
N_{c l}=t \sigma_{c l}=\frac{E t^{2}}{R \sqrt{3\left(1-v^{2}\right)}}
$$


Published in: Computers and Structures, 133C, 90-102.

DOI: http://dx.doi.org/10.1016/j.compstruc.2013.11.012

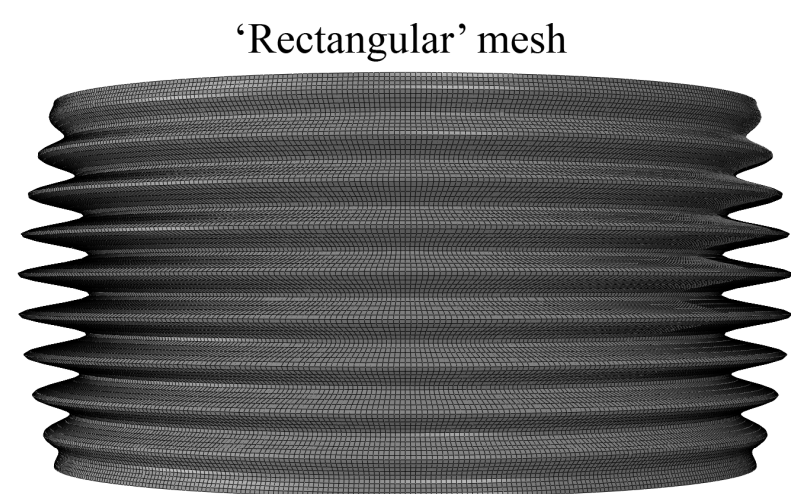

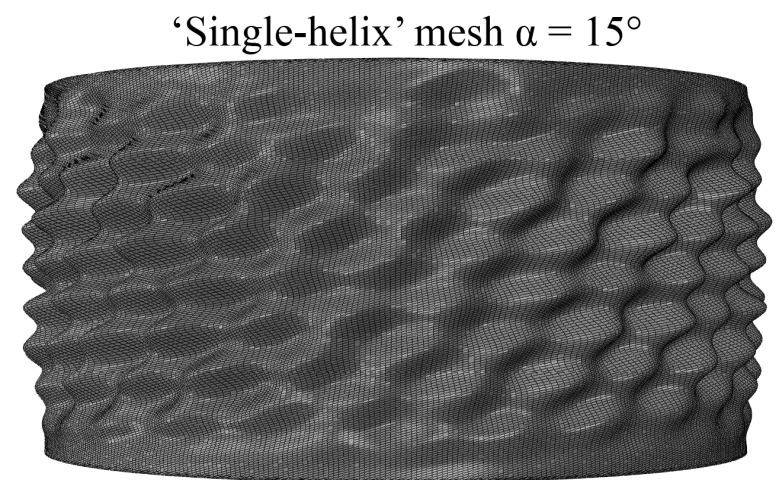

'Single-helix' mesh $\alpha=30^{\circ}$

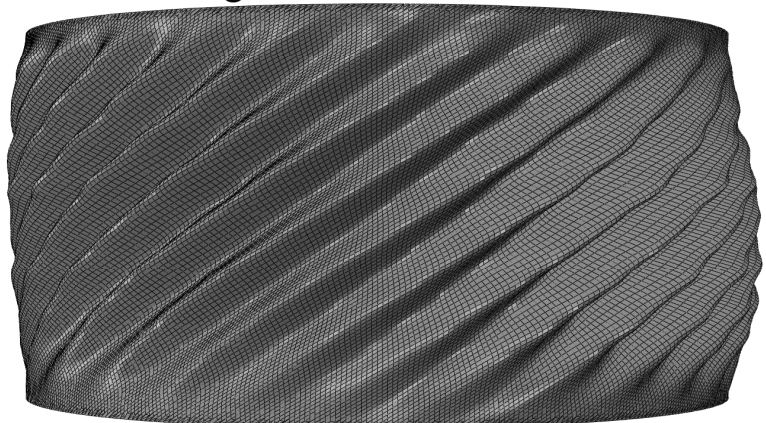

'Single-helix' mesh $\alpha=45^{\circ}$

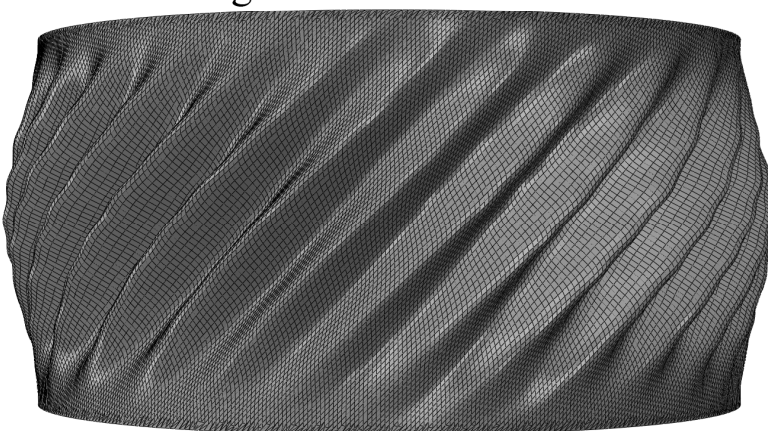

'Double-helix' mesh $\alpha=15^{\circ}$

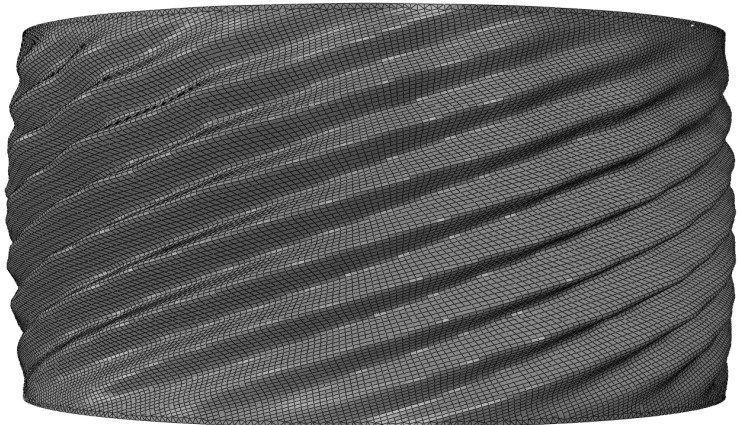

'Double-helix' mesh $\alpha=30^{\circ}$

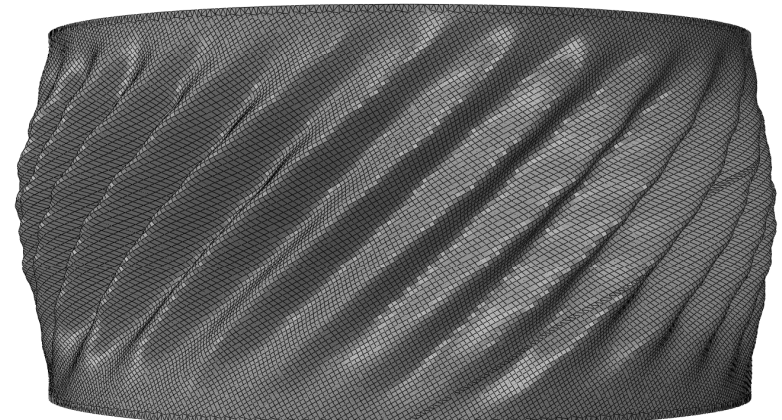

'Double-helix' mesh $\alpha=45^{\circ}$

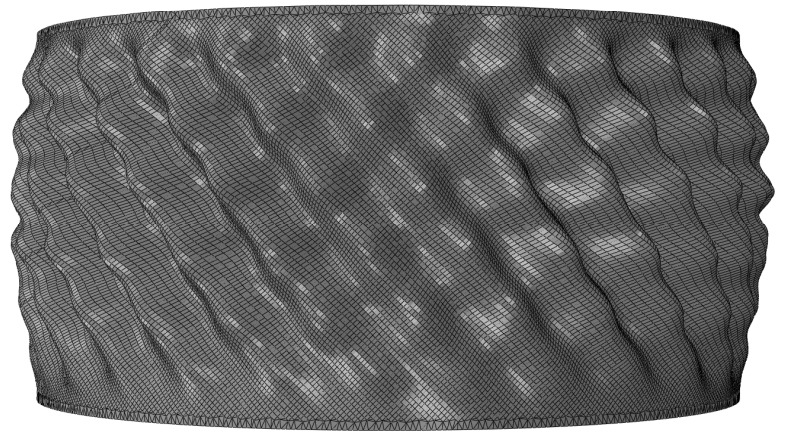

Fig. 8 - Linear bifurcation modes for a cylinder under axial compression meshed with different helical meshes (all critical loads within $1 \%$ of the classical solution $N_{c l}$ )

A set of linear bifurcation analyses were performed first to illustrate the effect of helical meshes on the critical bifurcation eigenmode. The analyses were performed on a 'rectangular' mesh first, followed by 'single-helix' and 'double-helix' meshes with helical angles of 15, 30 and 45 degrees. It should be noted that the axially compressed 
Published in: Computers and Structures, 133C, 90-102.

DOI: http://dx.doi.org/10.1016/j.compstruc.2013.11.012

cylinder exhibits many near-simultaneously critical eigenmodes within $1 \%$ of the classical value $N_{c l}[46,47]$ whose circumferential and axial wavelengths are related by the Koiter circle [19,37]. For the cylinder with a regular rectangular mesh, the critical mode was found to be axisymmetric ( $n=0$ circumferential full waves) with $m=18$ meridional half-waves. However, a simple characterisation of the predicted critical modes is more difficult for the helically-meshed cylinders (Fig. 8) because the helical mesh itself acts as a very minor imperfection which biases the orientation of the critical mode to lie more favourably with respect to the axis of the helix.

The same cylinder was next analysed with a series of geometrically nonlinear analyses to illustrate the effect of inclined helical meshes on the predicted nonlinear buckling load and the post-buckling path. Such a cylinder exhibits a discontinuous load path due to sudden bifurcation at buckling [40] which must be transformed into a smooth snapthrough response if it is to be followed by path-tracing methods. This is usually achieved by introducing a small mesh perturbation in the form of an eigenmode [48,49,50], though load-disturbance methods are also possible [51,52]. Using software such as ABAQUS or ANSYS, it is relatively simple to import an eigenmode from a linear bifurcation analysis as an initial imperfection, but only if the same mesh is retained. If not, significant errors may arise during interpolation of the nodal coordinates, especially if a mode computed with a regular rectangular mesh is applied to a helical mesh. The goal here was to perturb the cylinder into the same mode shape irrespective of the mesh orientation, so the perturbation had to be applied manually during the meshing procedure.

Geometrically nonlinear analyses were chosen to be damped quasi-static and displacement-controlled following the detailed recommendations of Kobayashi et al. [50]. Artificial damping introduces volume-proportional viscous forces into the structure which help to dissipate the strain energy released during buckling and allow for the efficient modelling of systems which exhibit local instabilities far into the postbuckling region within a static analysis. The viscous forces $F_{v}=c \mathbf{M}^{*} v$ are implemented in the global equilibrium equation as $P-I-F_{v}=0$, where $P$ and $I$ are the external and internal force vectors respectively, $c$ is the artificial damping factor, $\mathbf{M}^{*}$ is an artificial mass matrix calculated with unit density and $v$ is the vector of nodal velocities (calculated using a fictional time increment) [21]. The damping factor $c$ was 
Published in: Computers and Structures, 133C, 90-102.

DOI: http://dx.doi.org/10.1016/j.compstruc.2013.11.012

taken as $10^{-4}$, lower than the default value of $2 \times 10^{-4}$, so as to avoid excessive damping which may influence the highly imperfection sensitive post-buckling path.

Each mesh was perturbed into a mode with $n=23$ circumferential full waves and $m=$ 14 meridional half waves (Fig. 9). The perturbation amplitude was taken as $\delta / t=0.01$, suitable for the analysis of a nearly perfect shell. To characterise the mesh perturbation parametrically, the circumferential form of the eigenmode was expressed as a sine function while the meridional form was parametrised as a piecewise-continuous spline function $S(z)$ using the Matlab Curve Fitting toolbox, though a Fourier series characterisation could also be used. For a 'rectangular' mesh, the nodal coordinates were generated directly using Eqs 3 to 5 assuming:

$r(\theta, z)=R-\delta \sin (n \theta) S(z)$

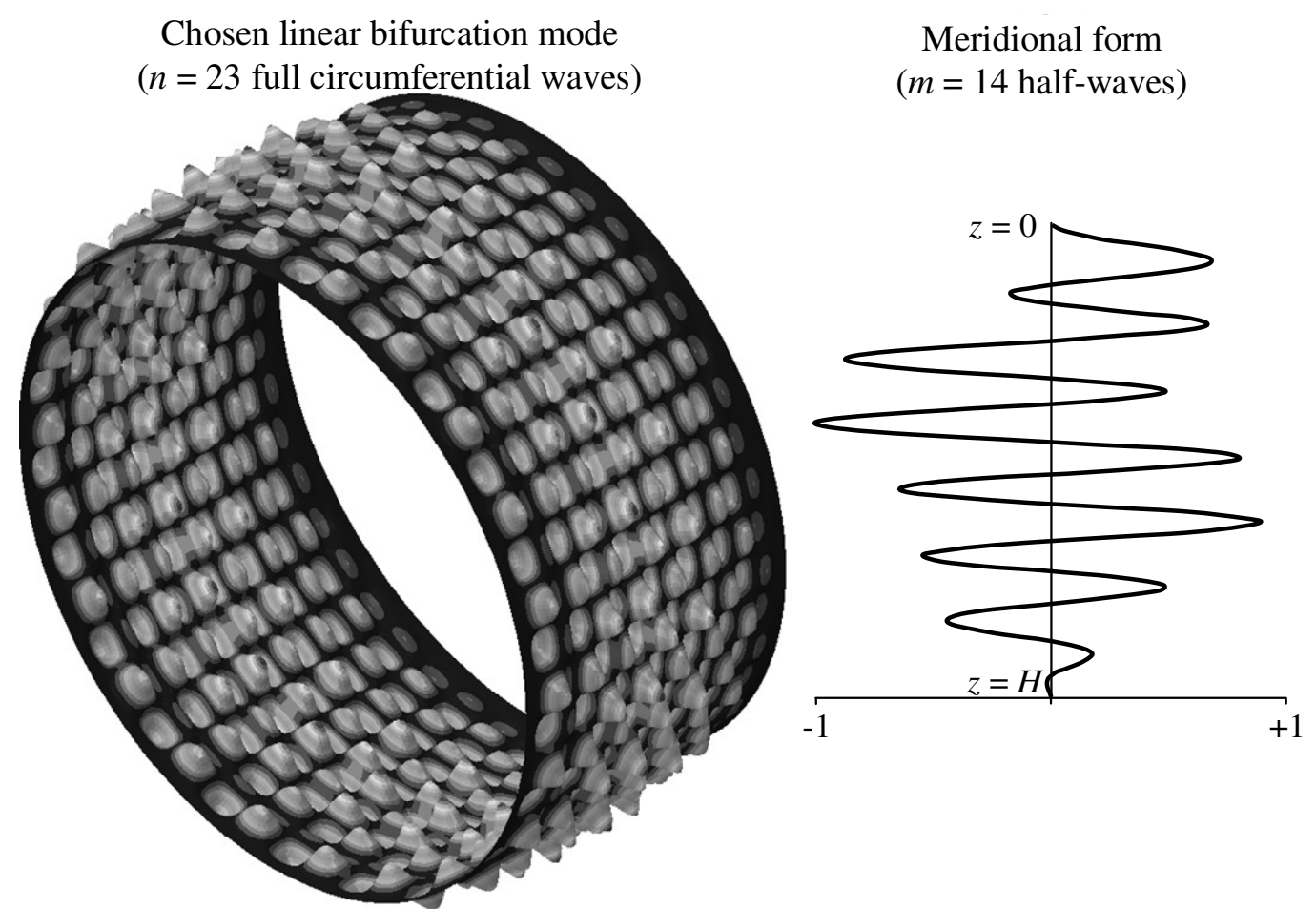

Fig. 9 - Shape of mesh perturbation in the form of a linear bifurcation eigenmode, with assumed amplitude $\delta / t=0.01$ (computed using a 'rectangular' mesh)

It is well known that post-buckling deformations and bifurcations of this structure are strongly dependent on the mode into which the cylinder was initially perturbed $[40,42,46,48,49,50,52,53]$. However, the exact form of the eigenmode perturbation shown in Fig. 9 is significant only insofar as it permits a clear illustration of the 
Published in: Computers and Structures, 133C, 90-102.

DOI: http://dx.doi.org/10.1016/j.compstruc.2013.11.012

influence of the helical meshing procedure on the predicted nonlinear post-buckling path. It has been verified that although a different choice of initial eigenmode perturbation pattern leads to a different post-buckling response, it is affected by helical meshing in a similar way.

The nodal coordinates of a helical mesh may be generated by replacing $r(\theta, z)$ in Eq. 20 with $r(\varphi, \zeta)$ or $r(\chi, \eta), z$ with $z(\varphi, \zeta)$ or $z(\chi, \eta)$ and $\theta$ with $\theta(\varphi, \zeta)$ or $\theta(\chi, \eta)$ as required (Eqs 8, 9, 13 and 14). An additional reference computation was performed on the regular rectangular mesh using the modified arc-length method [53] to follow the equilibrium path as far as possible into the post-buckling zone. The cylinder was loaded by an imposed axial displacement along one edge with the equivalent axial force at every increment being deduced from the nodal reactions at the other edge.

Table 1 - Nonlinear buckling loads for the axially-compressed cylinder

\begin{tabular}{|c|c|c|c|}
\hline Mesh type & Static Analysis & Helical angle $\alpha$ & Buckling load \\
\hline 'Rectangular' & Riks & $0^{\circ}$ & $0.899 N_{c l}$ \\
\hline 'Rectangular' & Damped & $0^{\circ}$ & $0.949 N_{c l}$ \\
\hline 'Single-helix' & Damped & $15^{\circ}$ & $0.975 N_{c l}$ \\
\hline 'Single-helix' & Damped & $30^{\circ}$ & $0.969 N_{c l}$ \\
\hline 'Single-helix' & Damped & $45^{\circ}$ & $0.987 N_{c l}$ \\
\hline 'Double-helix' & Damped & $15^{\circ}$ & $0.955 N_{c l}$ \\
\hline 'Double-helix' & Damped & $30^{\circ}$ & $0.956 N_{c l}$ \\
\hline 'Double-helix' & Damped & $45^{\circ}$ & $0.962 N_{c l}$ \\
\hline
\end{tabular}

The computed buckling loads are summarised in Table 1. The reference Riks analysis predicts the buckling strength of a 'perfect' shell to be approximately $0.90 N_{c l}$, corresponding closely to the analytical solution for the $\mathrm{C} 3$ boundary condition for $Z=$ 1000 [40]. The damped static analyses overshoot this value by around 5\% due to inertia effects [50]. Buckling loads calculated in this way should therefore be treated with care. The predictions are higher for a rotated helical mesh than a regular one, and are also higher for a 'single-helix' mesh than a 'double-helix' one. This may be understood in terms of the Rayleigh-Ritz principle [30], since mesh rotation leads to an over-constrained and excessively stiff system which requires higher strain energy to 
Published in: Computers and Structures, 133C, 90-102.

DOI: http://dx.doi.org/10.1016/j.compstruc.2013.11.012

develop a particular buckling mode shape, and thus a higher buckling load. This effect is more pronounced for the 'single-helix' mesh due to the significant additional element distortion. However, both types of helical mesh perform especially badly when $\alpha=45^{\circ}$ because at this angle the element distortion is greatest for the 'single-helix' mesh, whereas the inclined elements of the 'double-helix' mesh become subject to pure shear. In a previous study [29], the authors showed that the orientation of a mesh of rectangular elements with respect to the principal stress axes can play a significant role in the quality of the solution, with elements subject directly to pure shear exhibiting a poorer rate of convergence.

The calculated nonlinear load-displacement paths are illustrated in Figs 10 and 11 for the 'single-helix' and 'double-helix' meshes respectively. For reference, the Riks analysis of the rectangular cylindrical mesh is also shown. This is probably the best estimate of the true nonlinear response. It predicts that the cylinder undergoes a series of post-bucking bifurcations into modes with successively smaller numbers of circumferential waves, a mechanism that has been studied extensively elsewhere $[40,46,48,49,50,52,54]$ and is not the focus of the present discussion. The sharp reversal in the slope of the load path at every bifurcation indicates that it is likely to be difficult to trace the unstable post-buckling path using a path-following method $[48,53]$. Such algorithms often struggle to distinguish between closely-adjacent equilibrium paths and the analysis may either fail to converge, become stuck in an infinite loop that jumps back and forth between two paths or begin to trace a spurious load path due to accumulated round-off errors (especially deep in the post-buckling range after several thousand increments [42]). Indeed, despite considerable efforts, it was not possible to achieve numerical convergence beyond the fourth post-buckling bifurcation (marked with an '!', Figs 10 and 11). It is clear that even advanced commercial solvers currently struggle to trace such complex mode-jumping in thinwalled cylinders reliably using static analyses. Specialised methods for accurate detection of bifurcations and post-bifurcation paths have been devised in terms of hybrid static-dynamic procedures [48], catastrophe theory [63] and enhanced pathtracing using higher order derivatives of the tangent stiffness matrix or fold lines [64,65,66,67], amongst others, and have made much progress in overcoming this difficulty. Unfortunately, these highly specialised and complex procedures currently remain confined to the realm of personal custom-written finite element codes and are 
Published in: Computers and Structures, 133C, 90-102.

DOI: http://dx.doi.org/10.1016/j.compstruc.2013.11.012

usually not implemented in commercial software used by most analysts, designers and researchers.

A damped static analysis using a 'rectangular' mesh $\left(\alpha=0^{\circ}\right)$ predicts that the cylinder will jump onto a stable equilibrium path (marked with an 'A'; see also Fig. 12) directly underneath the initial bifurcation point, this load path having also been located by the Riks analysis. Thereafter it undergoes several further mode jumps before settling onto what appears to be a final stable path at a load of approximately $0.25 N_{c l}$ (marked with a 'B'; Fig. 12), corresponding quite well to both experimental and theoretical results for a cylindrical shell with $Z=1000$ (see Figs 3.52e and 3.61e of Yamaki [40]). There is no doubt that the Riks analysis too would have eventually settled onto this stable path had the analysis been able to follow the numerous intermediate bifurcations. Most importantly, these equilibrium paths illustrate that the helical meshes have trouble in accurately modelling the post-buckling behaviour, although every analysis eventually settled onto essentially the same stable path 'B' and exhibited the same post-buckling mode. The transition between the initial bifurcation point and the path 'B' suggests that the helically meshed cylinder behaves like an imperfect structure, since imperfections are known either to smooth or even to eliminate bifurcation points entirely [40]. For the helical meshes, the 'single-helix' mesh with $\alpha=15^{\circ}$ and the 'double-helix' mesh with $\alpha=45^{\circ}$ appear to produce quite significant imperfections. That a mere rotation of the mesh causes imperfections that are sufficiently serious to achieve such a change in the post-buckling behaviour illustrates the fact that helical meshing is not well suited to the analyses of perfect shells. 
Published in: Computers and Structures, 133C, 90-102.

DOI: http://dx.doi.org/10.1016/j.compstruc.2013.11.012

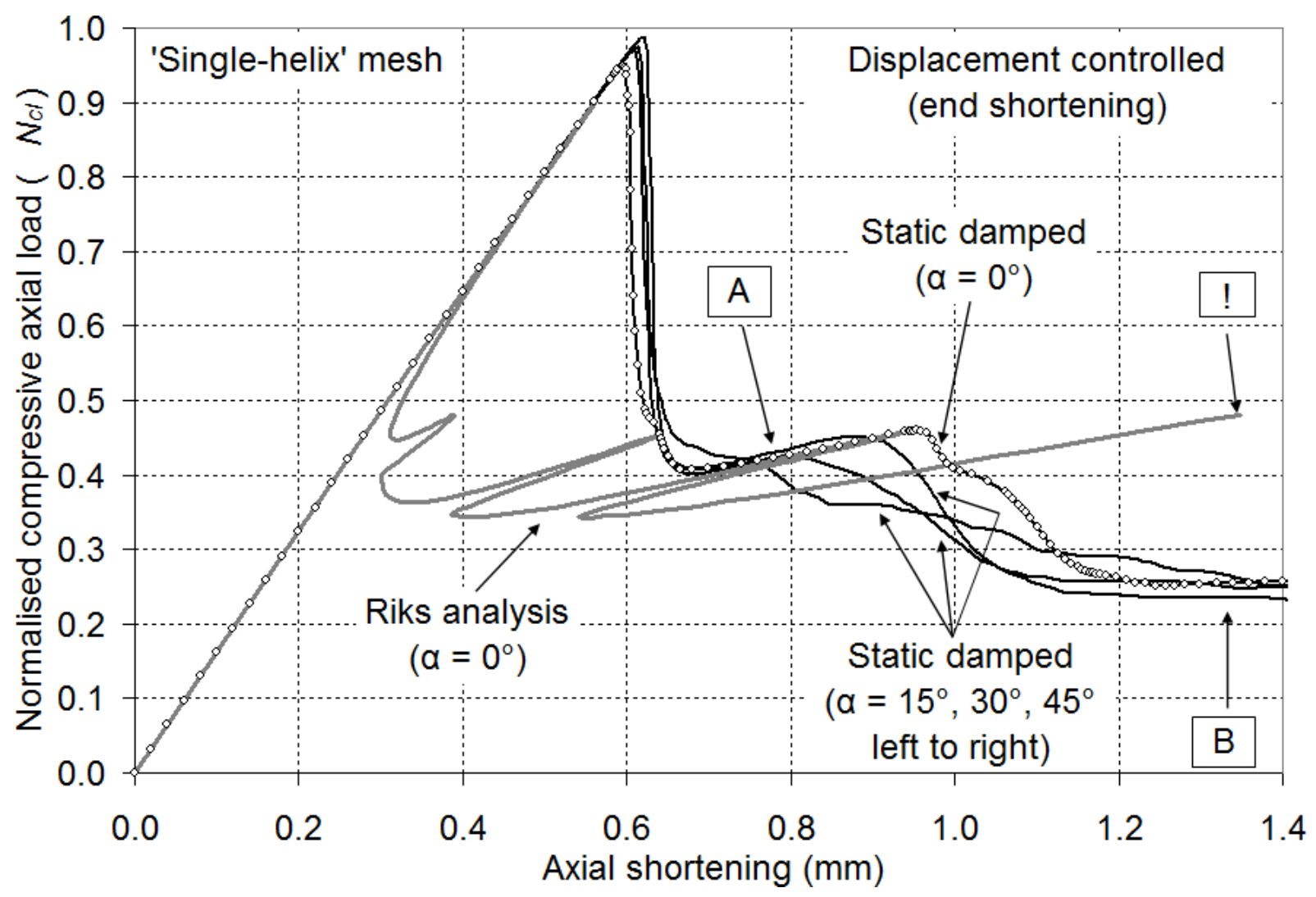

Fig. 10 - Nonlinear equilibrium paths for an axially-compressed cylinder with 'singlehelix' meshes of varying helical angles 
Published in: Computers and Structures, 133C, 90-102.

DOI: http://dx.doi.org/10.1016/j.compstruc.2013.11.012

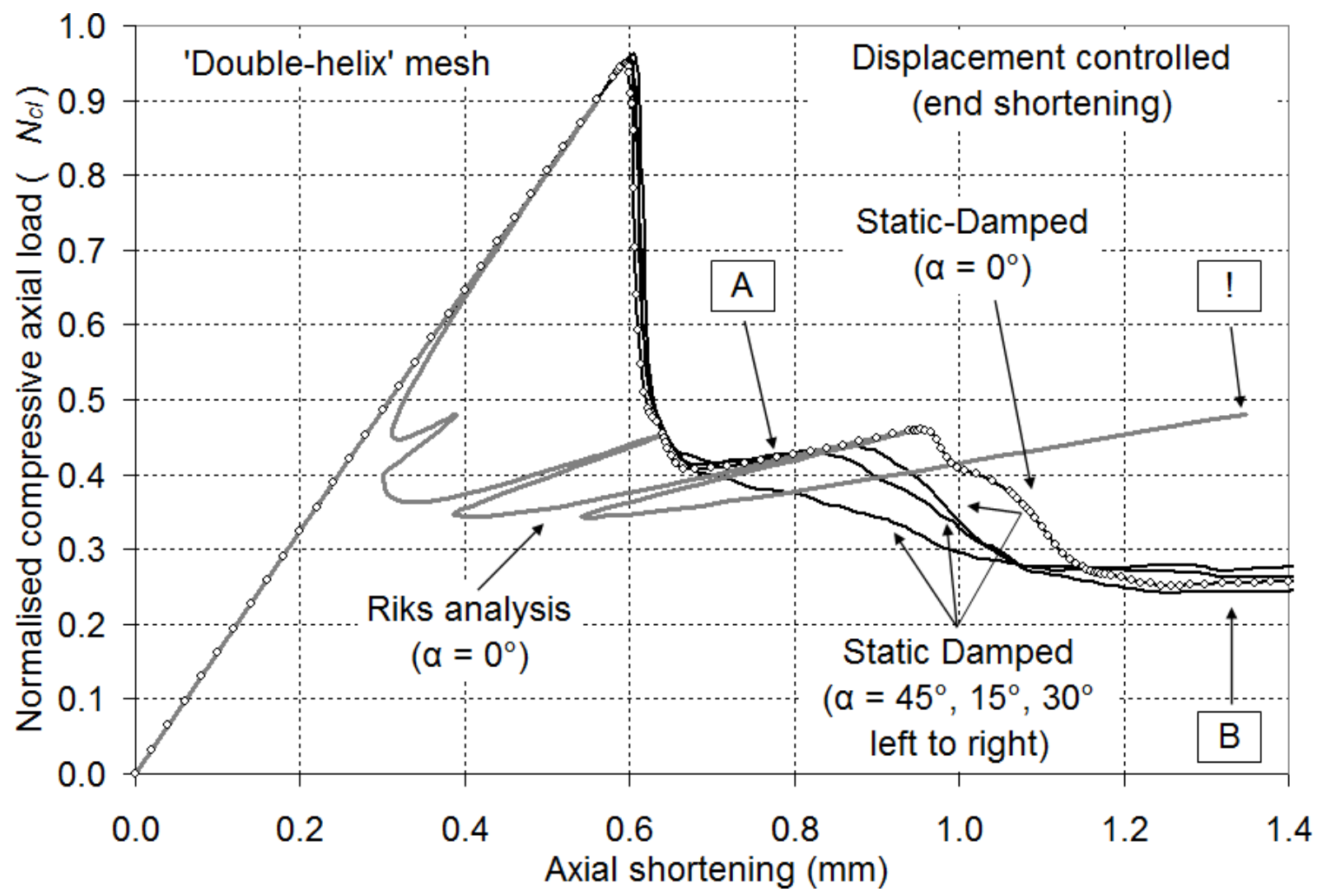

Fig. 11 - Nonlinear equilibrium paths for an axially-compressed cylinder with 'doublehelix' meshes of varying helical angles

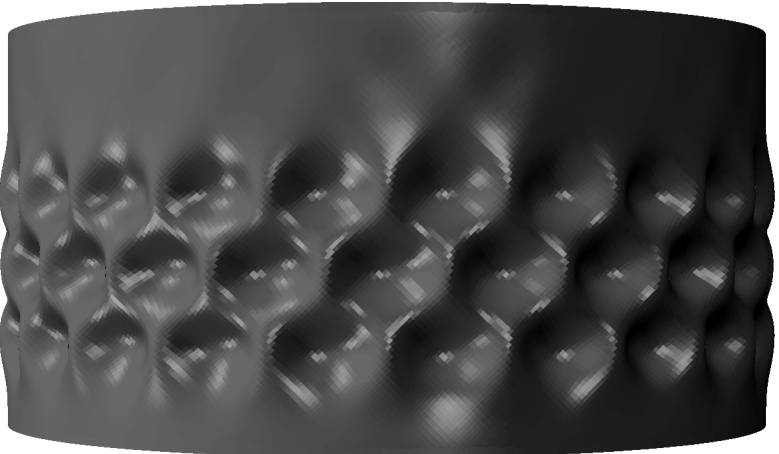

a) Point $\mathrm{A}$ in Figs $10 \& 11$

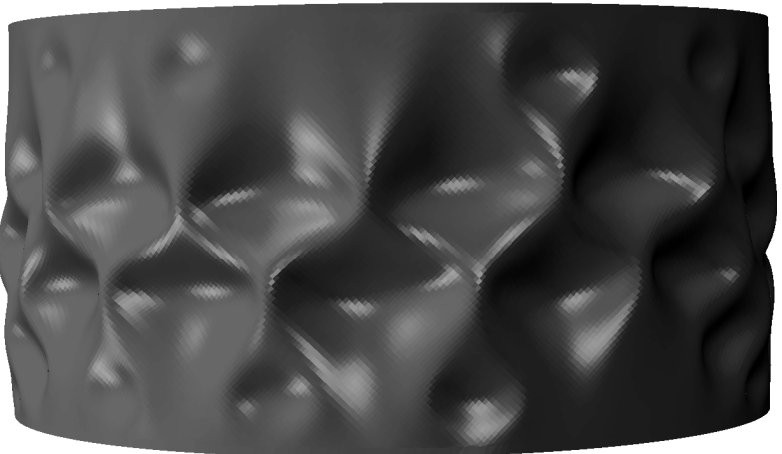

b) Point B in Figs $10 \& 11$

Fig. 12 - Selected post-buckling deformed modes for the axially-compressed cylinder

\section{Behaviour of spiral welded cylinders under axial compression}

The following example of an idealised spiral welded cylinder under uniform axial compression illustrates the intended implementation of the helical meshing procedure. Deviations of the shell mid-surface lead to localisation of the stresses even under uniform load distributions which, in turn, localises the buckling mode to be in the vicinity of the imperfection. Systems with locally elevated compressive stresses thus 
Published in: Computers and Structures, 133C, 90-102.

DOI: http://dx.doi.org/10.1016/j.compstruc.2013.11.012

exhibit a significantly decreased imperfection sensitivity [61,62]. In particular, the minute numerical 'imperfections' introduced by the helical meshing become negligible in comparison to the effect of explicitly modelled geometric imperfections and the analysis of the structure may now benefit fully from the well-defined geometrical representation.

Large cylindrical shells such as silos, tanks and butt-welded pipelines are constructed by welding together individual strakes of rolled sheet metal. The strake edges are slightly curled due to the rolling process and the shrinking of the circumferential weld during cooling [55]. The result is a regular axisymmetric depression oriented towards the interior of the shell $[41,46]$. Two rationally-based and idealised mathematical characterisations of the shape of this depression were proposed by Rotter and Teng [55]. This has since become one of the most common and probably most deleterious realistic imperfection forms for thin-walled hollow cylinders under axial compression $[41,56,57,58,59,60]$. Known as the 'axisymmetric weld depression', it is defined using the present notation as:

$r(\theta, z)=R-\delta e^{-\pi\left|z-z_{w}\right| \lambda}\left(\cos \frac{\pi\left|z-z_{w}\right|}{\lambda}+\sin \frac{\pi\left|z-z_{w}\right|}{\lambda}\right)$

where $\lambda=\frac{\pi \sqrt{R t}}{\left[3\left(1-v^{2}\right)\right]^{0.25}}$

In the above, $\lambda$ is the linear axisymmetric meridional bending half-wavelength from classical shell theory $[15,16,17,18,19], z_{w}$ is the $z$-coordinate of the weld depression assuming the range $0 \leq z \leq H$ and $\delta$ is the imperfection amplitude. The bending halfwavelength is assumed to control the extent of the penetration of the depression into the wall of the cylinder, and the imperfection has effectively vanished after a distance of approximately $\lambda$ from $z_{w}$, due to the decaying exponential term.

Full-scale measurements of the imperfect geometry of spiral welded tubes are difficult to obtain and, to the best of the authors' knowledge, there have not yet been any published attempts to characterise measured imperfections in a manner similar to Eq. 21 above. However, the method of manufacturing spiral welded tubes (Fig. 1) subjects the rolled sheet metal to deformations that are similar to those of circumferential welding, although in a different plane. Thus it is likely that the process results in a 
Published in: Computers and Structures, 133C, 90-102.

DOI: http://dx.doi.org/10.1016/j.compstruc.2013.11.012

regular inward depression of the wall of the tube that is orthogonal to and follows the path of the spiral weld. For want of better information, and to illustrate the intended capabilities of the methods of helical meshing presented in this paper, Eq. 21 was adopted directly for the rectangular image $\varphi-\zeta$ or $\chi-\eta$ planes so that an 'axisymmetric' weld depression defined in terms of $\zeta$ or $\eta$ maps into a spiral weld depression in the $x$ $y-z$ space. The weld was positioned through the middle of the strip at $\zeta=1 / 2 P$ (Eq. 1 , Fig. 3) or $\eta=1 / 2 \rho$ (Eq. 15, Fig. 4) and thus away from the strip misalignment (Figs 5 and 6). A spiral weld defined on the $\varphi-\zeta$ plane (Eq. 23) for the 'single-helix' mesh is oriented orthogonally to the vertical axis in the $x-y-z$ space due to the non-conformal transformation, whereas a weld defined on the $\chi-\eta$ plane (Eq. 24) for the 'double-helix' mesh is fully orthogonal to the path of the spiral due to the conformal transformation. The spiral weld depression imperfection is illustrated in Fig. 13, where it was assumed for simplicity that $\lambda_{1}=\lambda_{2}=\lambda$ (Eq. 22).

'Single-helix' mesh: $r(\varphi, \zeta)=R-\delta e^{-\pi|\zeta-1 / 2 P| / \lambda_{1}}\left(\cos \frac{\pi|\zeta-1 / 2 P|}{\lambda_{1}}+\sin \frac{\pi|\zeta-1 / 2 P|}{\lambda_{1}}\right)$

'Double-helix' mesh: $r(\chi, \eta)=R-\delta e^{-\pi|\eta-1 / 2 \rho| \lambda_{2}}\left(\cos \frac{\pi|\eta-1 / 2 \rho|}{\lambda_{2}}+\sin \frac{\pi|\eta-1 / 2 \rho|}{\lambda_{2}}\right)$

Cylindrical tube with exaggerated spiral imperfection - 'double-helix' mesh for illustration purposes only

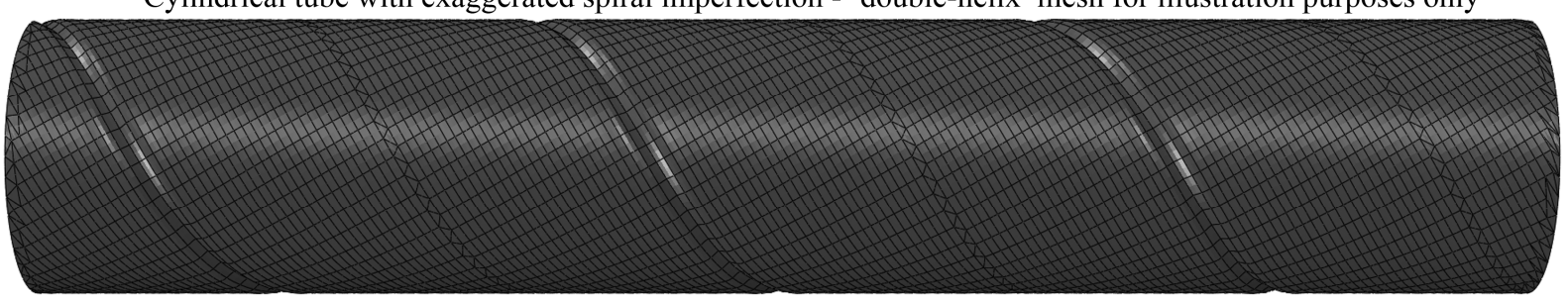

a) 'Single-helix' mesh spiral weld detail

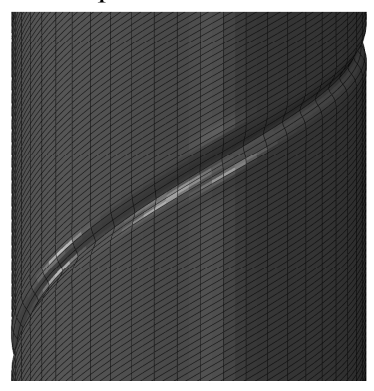

b) 'Double-helix' mesh spiral weld detail

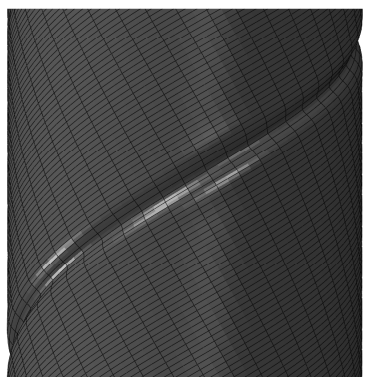

c) 'Rectangular' mesh axisymmetric weld detail

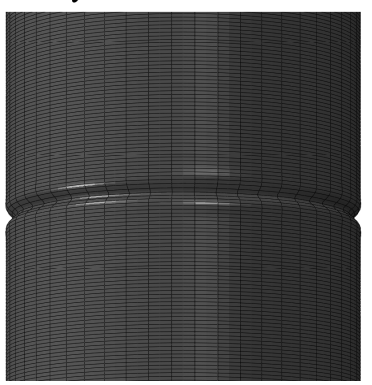

Fig. 13 - Modelling of spiral weld imperfections in cylindrical tubes 
Published in: Computers and Structures, 133C, 90-102.

DOI: http://dx.doi.org/10.1016/j.compstruc.2013.11.012

A parametric finite element study was performed to explore the nonlinear elastic buckling behaviour of an axially-compressed thin-walled cylinder $(R / t=1000)$ with spiral weld imperfections of different amplitudes as defined by Eqs 22 and 23 above. The shell was loaded with $N_{c l}$ (Eq. 19) along one edge which was restrained against radial displacement only (simply-supported S3 condition) while the other edge was restrained against both radial and meridional displacement (simply-supported S1 condition). The equilibrium path was followed with the modified arc-length method [53] until just after the first bifurcation or limit point.

The authors' custom-written software was used to generate models of the cylinder with 'single-helix' and 'double-helix' meshes which were then analysed using ABAQUS/Standard v. 6.10.1 [21]. Two helical angles were investigated; $\alpha=15^{\circ}$ (shallow) and $45^{\circ}$ (steep). The length of the shell was chosen as 3 complete revolutions of the helix, leading to a length to diameter ratio of $H / D$ of 2.53 and 9.43 for $\alpha=15^{\circ}$ and $45^{\circ}$ respectively. The elements were specified to be nine-node reduced-integration thin shell elements with 5 dofs per node (S9R5) shell elements together with six-node triangular shell elements (STRI65) as 'filler' for the end boundaries. The aspect ratio of the quadrilateral elements was approximately 2 while the shortest side of the element was limited to $0.5(R t)^{1 / 2}$ or $0.2 \lambda$ (Eq. 22) to ensure that meshes were sufficiently fine to model the severe local bending associated with buckling.

The imperfection amplitude $\delta / t$ of the spiral welds was varied from 0 to 3 , representative of practical cylindrical structures under axial compression $[43,46]$. The imperfection sensitivity relationship of the predicted buckling strength against imperfection amplitude is shown in Fig. 14, together with comparable data for the axisymmetric weld depression (Eq. 21 and [55]). The original analysis of the axisymmetric weld depression showed that an imperfection amplitude of just half a wall thickness led to buckling strengths of only $40 \%$ of $N_{c l}$, which is a considerable loss of strength. The results presented here indicate that the assumed form of the spiral weld imperfection may be just as damaging to the strength of an axially-compressed cylinder. A number of additional observations may also be made. 
Published in: Computers and Structures, 133C, 90-102.

DOI: http://dx.doi.org/10.1016/j.compstruc.2013.11.012

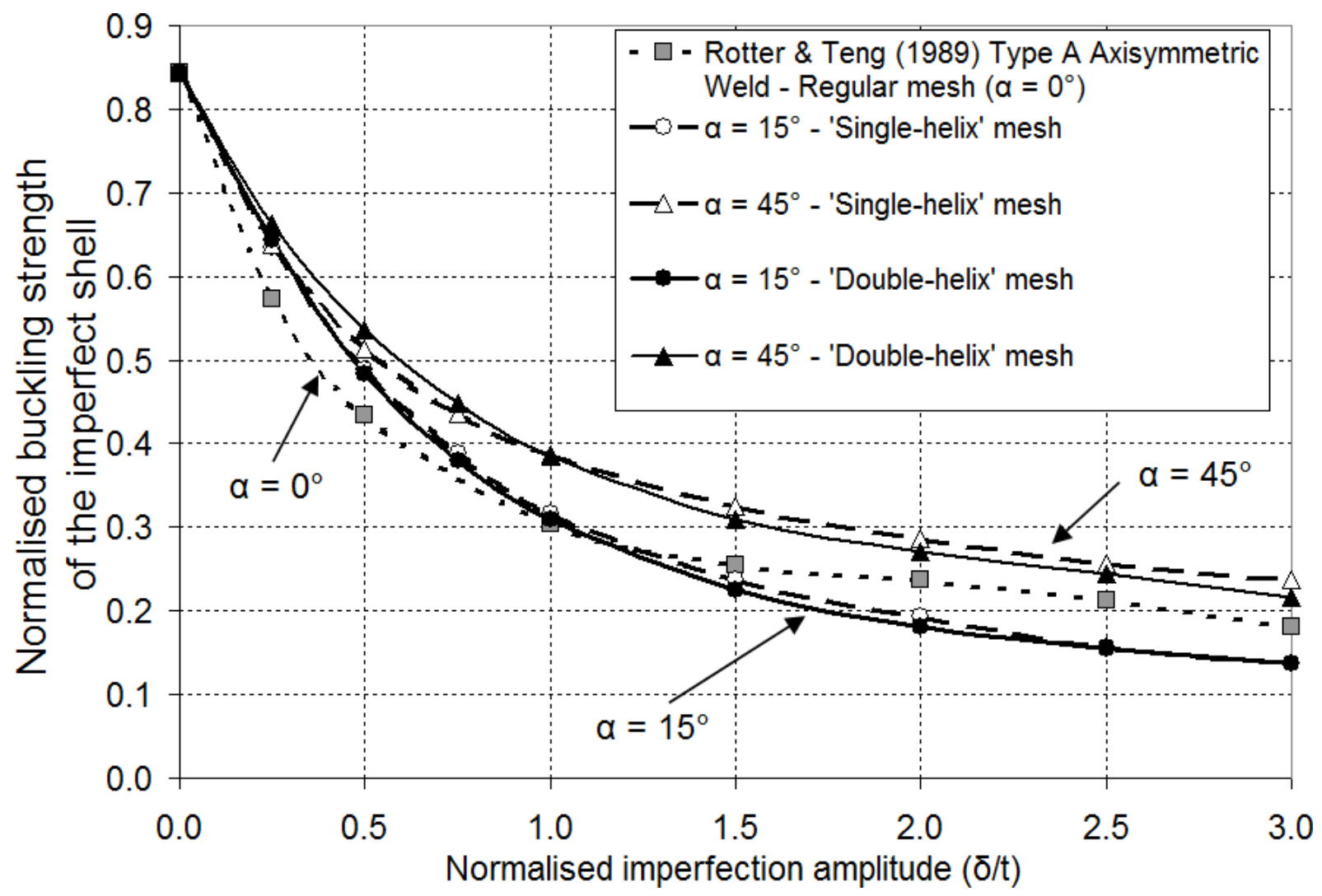

Fig. 14 - Variation of buckling strength with helical weld depression amplitude

First, the buckling strength of the 'perfect shell' is only approximately $0.843 N_{c l}$ in the present analysis, due to pre-buckling bending deformations associated with the S3 boundary condition at the loaded edge. This value corresponds closely to the analytical solution for a medium length axially-compressed cylinder [40]. Second, the spiral imperfection spans the full length of the cylinder and is inevitably present at both end boundaries (Fig. 13), so that the buckling modes always formed near the loaded edge, regardless of imperfection amplitude and helix angle. Deeper imperfection amplitudes caused the buckling mode to penetrate further into the shell (Figs 15 and 16) and the buckles are clearly orientated normal to the spiral weld.

Third, the 'single-helix' mesh leads to very slightly lower predicted strengths than the 'double-helix' mesh at small imperfection amplitudes $(\delta / t<1.0)$. This trend is reversed at larger imperfection amplitudes. Indeed, the type of mesh transformation (i.e. nonconformal or conformal) appears to have only a minor effect on both the buckling strength and the shape of the incremental buckling mode. More importantly, for both types of helical mesh, the steeper helix angle $\alpha=45^{\circ}$ leads to significantly higher 
Published in: Computers and Structures, 133C, 90-102.

DOI: http://dx.doi.org/10.1016/j.compstruc.2013.11.012

predicted buckling strengths than the shallower angle $\alpha=15^{\circ}$. This is because the effective wavelength of the spiral weld imperfection along any axial generator increases with the helix angle, which in turn leads to a higher buckling strength under axial compression. The mechanics of this behaviour were described in detail in Teng and Rotter [41].
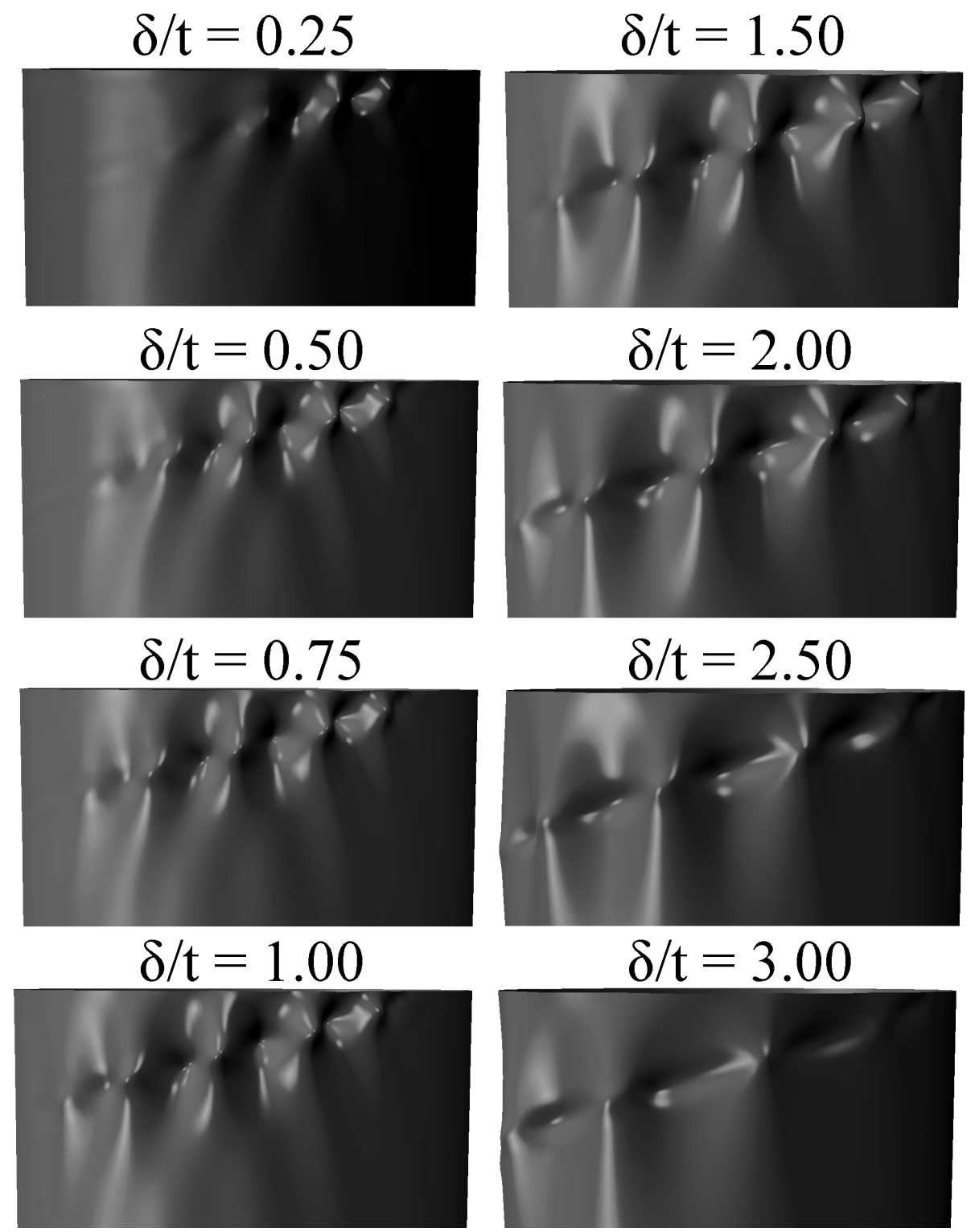

Fig. 15 - Incremental buckling mode for an imperfect cylinder with spiral welds at a helical angle of $\alpha=15^{\circ}$ ('double-helix' mesh). The top of each figure is at the loaded boundary of the cylinder. 
Published in: Computers and Structures, 133C, 90-102.

DOI: http://dx.doi.org/10.1016/j.compstruc.2013.11.012

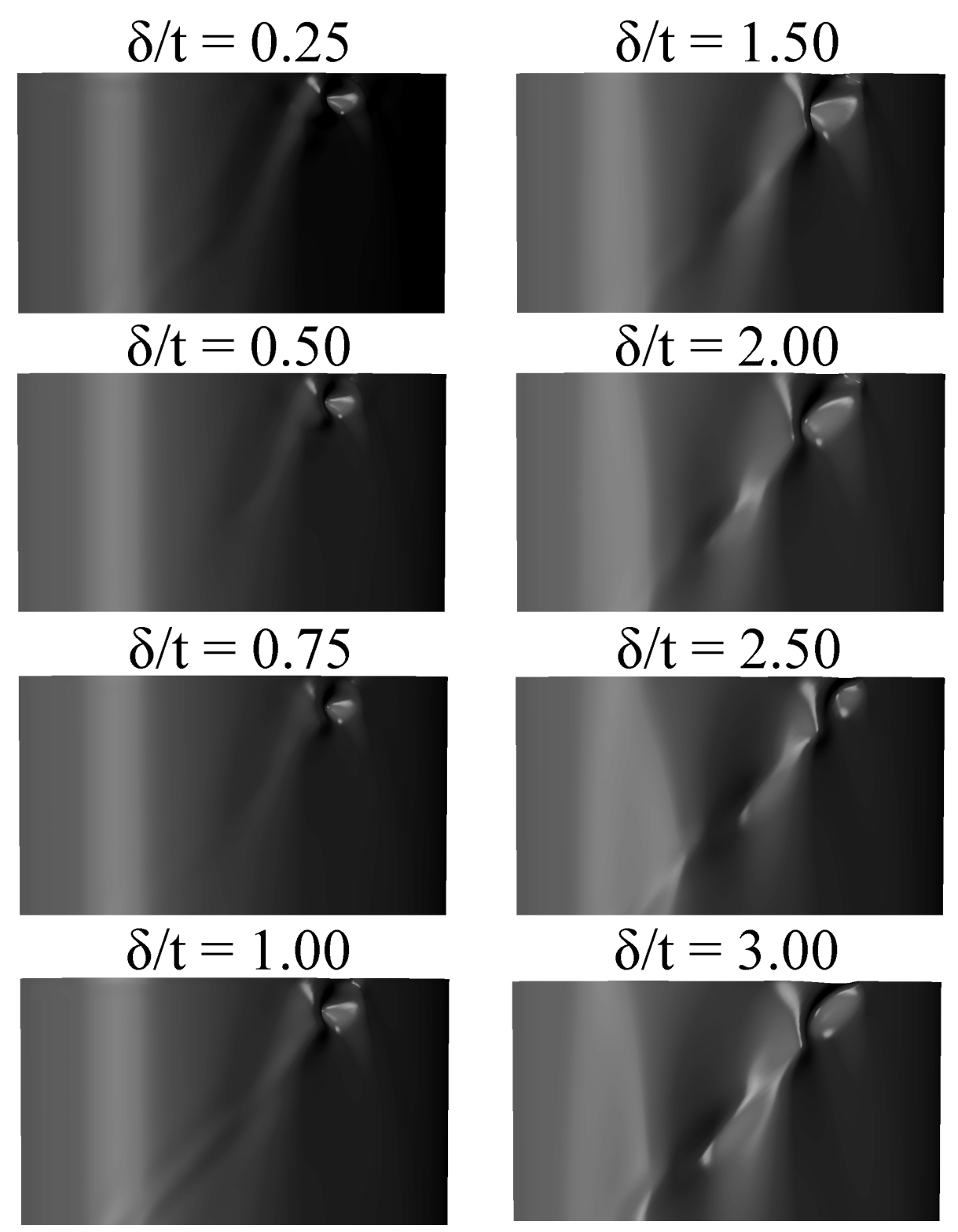

Fig. 16 - Incremental buckling mode for an imperfect cylinder with spiral welds at a helical angle of $\alpha=45^{\circ}$ ('double-helix' mesh). The top of each figure is at the loaded boundary of the cylinder.

\section{Conclusions}

This paper has presented an introductory study of the structural behaviour of thin cylindrical shells with helical features, focusing on the behaviour of the axiallycompressed spiral wound cylinder.

A robust and highly general computational method has been proposed to generate complete meshes of well-structured inclined quadrilateral finite elements. Named 'helical meshing', it allows high quality orthogonal meshes to be oriented at an optimal 
Published in: Computers and Structures, 133C, 90-102.

DOI: http://dx.doi.org/10.1016/j.compstruc.2013.11.012

inclination relative to the spiral feature and eliminates the need for freely meshed triangulations. In particular, geometric deviations, residual stresses, weld metal and other material-related features that are spiral in nature may now be modelled with great ease and accuracy.

The effect of helical meshing was demonstrated first on a perfect thin-walled cylinder under axial compression with an eigenmode perturbation. A series of nonlinear pathtracing elastic analyses showed that helical meshes are less accurate in modelling the post-buckling behaviour. This is because the element distortions, rotations and circular boundary triangulations associated with helical meshes constitute effective imperfections that are severe enough to eliminate post-buckling bifurcations, even in an otherwise nominally 'perfect' structure. For this reason, helical meshing is not appropriate modelling technique for imperfection-sensitive perfect cylindrical geometries.

A second example focused on geometrically imperfect spirally welded cylinders under axial compression. The mathematical characterisation of the spiral weld was adopted from a previous study of axisymmetric circumferential weld depressions. A set of nonlinear elastic buckling analyses showed that a spiral weld is detrimental to the strength of a cylindrical shell to essentially the same extent as an axisymmetric weld, though the effect does depend on the helix angle.

It is hoped that the methods of helical meshing presented in this paper will facilitate future studies in the computational modelling of cylindrical shells with spiral features. To understand the behaviour of these structures fully, more research is needed to better determine the types of imperfections and patterns of residual stresses that may be expected to occur in spiral welded tubes.

\section{Acknowledgements}

This work was carried out as part of the EU RFCS Combitube research project funded by the European Commission, grant contract RFSR-CT-2011-00034. 
Published in: Computers and Structures, 133C, 90-102.

DOI: http://dx.doi.org/10.1016/j.compstruc.2013.11.012

\section{References}

[1] Hanada M., Takeda H., Fukushima H., Koizumi I. \& Noguchi Y. (1986). "Development of high speed submerged arc welding in spiral pipe mill" Transactions ISIJ, 26, 433-438.

[2] Burstall T. (1997). "Bulk Water Pipelines" Thomas Telford Ltd.

[3] Hardenbergh W.A. (1945). "Water supply and purification" 2nd ed. International textbook company, Scranton, Pa.

[4] Liu H. (2003). "Pipeline Engineering" CRC Press.

[5] CUR (2005). "Handbook Quay Walls - Publication N. 211 E", Centre for Civil Engineering Research and Codes (CUR), Gouda, Holland.

[6] Lancaster J. (1997). "Handbook of structural welding: Processes, materials and methods used in the welding of major structures, pipelines and process plants." Woodhead Publishing.

[7] Radaj D. (1990). "Design and analysis of fatigue resistant welded structures." Woodhead Publishing.

[8] Andrews R.M. \& Pistone V. (2002). "European pipeline research group studies on ductile crack propagation in gas transmission pipelines." European Structural Integrity Society, 30, 377-384.

[9] ArcelorMittal (2011). "Spirally welded steel pipes" Corporate Brochure, ProjectsEurope, Foundation Solutions, The Netherlands.

[10] Gerwick B.C. Jnr (2007). "Construction of Marine and Offshore Structures" 3rd Ed., CRC Press.

[11] Bishop R.E.D. (1979). "Vibration" Cambridge University Press. 
Published in: Computers and Structures, 133C, 90-102.

DOI: http://dx.doi.org/10.1016/j.compstruc.2013.11.012

[12] Woodcock C.R. \& Mason J.S. (1987). "Bulk Solids Handling: An introduction to the practice and technology." Chapman \& Hall.

[13] Stephenson D. (1976). "Pipeline design for water engineers." Elsevier Scientific Publications, Amsterdam.

[14] AWWA (2004). "Steel Pile - A Guide for Design and Installation. Manual M11" 4th Ed. American Water Works Association.

[15] Flügge W. (1957). “Statik und Dynamik der Schalen” Springer-Verlag.

[16] Timoshenko S.P. \& Gere J.M. (1963). "Theory of elastic stability" McGraw-Hill International Editions.

[17] Novozhilov V.V. (1970). "Thin shell theory" Wolters-Noordhoff, Groningen.

[18] Seide P. (1975). "Small elastic deformations of thin elastic shells." Nordhoff, Leyden, Holland.

[19] Calladine C. R. (1983). "Theory of shell structures" Cambridge University Press.

[20] Dong P., Hong J.K. \& Leis B.N. (2006). "Computational simulation of line-pipe fabrication processes" Proceedings of the 6th International Pipeline Conference, September 25-29, Calgary, Canada.

[21] ABAQUS (2011). “ABAQUS Version 6.10.1 Theory Manual” Dassault Systèmes Simulia Corp., Providence, RI, USA.

[22] Wirth S. (2008). "Beulsicherheitsnachweise für schalenförmige Bauteile nach EN 1993-1-6" Doktor-Ingenieur Dissertation, Universität Duisburg-Essen.

[23] ANSYS (2007). “ANSYS Version 11.0.” ANSYS Inc., Canonsburg, PA, USA. 
Published in: Computers and Structures, 133C, 90-102.

DOI: http://dx.doi.org/10.1016/j.compstruc.2013.11.012

[24] Arif A.F.M., Al-Osmari A.S., Yilbas B.S. \& Al-Nassar Y.N. (2011). “Thermal stress analysis of spiral laser-welded tube" Journal of Materials Processing Technology, 211, 675-687.

[25] Ohnishi S., Ono K. \& Akiyama M. (2011). "Analytical study on seismic performance of hollow spiral steel pipes under cyclic loading" Procedia Engineering, 14, 898-905.

[26] Nishimura N., Ono K. \& Ikeuchi T. (1995). "A constitutive equation for structural steels based on a monotonic loading curve under cyclic loading." Doboku Gakkai Ronbunshu, 513, I-31, 27-38.

[27] Nishimura N., Ikeuchi T. \& Taniguchi N. (1998). "Numerical simulation on damage to pipe piers in Hyogo-ken Nanbu earthquake." Engineering Structures, 20(46), 291-299.

[28] Forouzan M.R., Mirfalah Nasiri S.M., Mokhtari A., Heidari A. \& Golestaneh S.J. (2012). "Residual stress prediction in submerged arc welded spiral pipes" Materials and Design, 33, 384-394.

[29] Sadowski A.J. \& Rotter J.M. (2013). "On the relationship between mesh and stress field orientations in linear stability analyses of thin plates and shells" Finite Elements in Analysis and Design, 73, 42-54.

[30] Cook R.D., Malkus D.S., Plesha M.E. \& Witt R.J. (2002). "Concepts and applications of finite element analysis" 4th edition. John-Wiley \& Sons.

[31] Zienkiewicz O.C. \& Taylor R.L. (2005) "The finite element method - For solid and structural mechanics". 6th edition, Elsevier.

[32] George P-L. \& Borouchaki H. (1998). "Delaunay Triangulation and Meshing Application to Finite Elements" Editions Hermes, Paris. 
Published in: Computers and Structures, 133C, 90-102.

DOI: http://dx.doi.org/10.1016/j.compstruc.2013.11.012

[33] Wang X.-L., Payzant E.A., Taljat B., Hubbard C.R., Keiser J.R. \& Jirinec M.J. (1997). "Experimental determination of the residual stresses in a spiral weld overlay tube." Materials Science and Engineering, A232, 31-38.

[34] Matlab (2004). "Matlab Version 7.0 R14" Mathworks, Natick, MA, USA.

[35] Kepner J. (2002). "Parallel Programming with MatlabMPI" (Matlab plug-in) Lincoln Laboratory, Massachusetts Institute of Technology, Lexington, MA, USA.

[36] Hjelle $\varnothing . \&$ Dæhlen M. (2006). "Triangulations and Applications" SpringerVerlag, Berlin-Heidelberg.

[37] Koiter W.T. (1945). "On the stability of elastic equilibrium." PhD Thesis, Delft University, Holland.

[38] Koiter W.T. (1963). "The effect of axisymmetric imperfections on the buckling of cylindrical shells under axial compression." Proc. Kon. Ned. Akad. Wet., B66, 265279. (See also Appl. Mech. Rev., 18, Review 3387, 1965).

[39] Hutchinson J.W. \& Koiter W.T. (1970). "Postbuckling theory" Applied Mechanics Reviews, 23, 1353-1366.

[40] Yamaki, N. (1984). "Elastic stability of circular cylindrical shells." North Holland, Amsterdam.

[41] Teng J.G. \& Rotter J.M. (1992). "Buckling of pressurised axisymmetrically imperfect cylinders under axial loads." ASCE Journal of Engineering Mechanics, 118(2), 229-247.

[42] Teng J.G. \& Song C.Y. (2001). "Numerical models for nonlinear analysis of elastic shells with eigenmode-affine imperfections." International Journal of Solids and Structures, 38, 3263-3280. 
Published in: Computers and Structures, 133C, 90-102.

DOI: http://dx.doi.org/10.1016/j.compstruc.2013.11.012

[43] EN 1993-1-6 (2007). "Eurocode 3: Design of Steel Structures, Part 1-6: Strength and Stability of Shell Structures." Comité Européen de Normalisation, Brussels.

[44] Donnell L.H. \& Wan C.C. (1950). "Effect of imperfections on buckling of thin cylinders and columns under axial compression." Journal of Applied Mechanics, ASME, 17(1), 73-83.

[45] Bushnell D. (1985). "Computerised buckling analysis of shells." Martinus Nijhoff Publishers, Dordrecht.

[46] Rotter J.M. (2004). "Buckling of cylindrical shells under axial compression." Buckling of thin metal shells, J.G. Teng and J.M. Rotter, eds., Spon, London, 42-87.

[47] Limam, A. (1991) "Flambage de coques cylindriques sous combinaison de chargement: pression interne et compression axiale." Thèse de doctorat, Institut National des Sciences Appliquées (INSA) de Lyon, France.

[48] Riks E., Rankin C.C. \& Brogan F.A. (1996). "On the solution of mode jumping phenomena in thin-walled shell structures." Comput. Methods Appl. Mech. Eng., 136, 59-92.

[49] Hong T. \& Teng J.G. (2008). "Imperfection sensitivity and postbuckling analysis of elastic shells of revolution." Thin-Walled Structures, 46, 1338-1350.

[50] Kobayashi T., Mihara Y. \& Fujii F. (2012). "Path-tracing analysis for postbuckling process of elastic cylindrical shells under axial compression." Thin-Walled Structures, 61, 180-187.

[51] Chan A.S.L. \& Trbojevic V.M. (197). "Thin shell finite element by the mixed method formulation - Parts 2 and 3." Computer Methods in Applied Mechanics and Engineering, 10, 75-103. 
Published in: Computers and Structures, 133C, 90-102.

DOI: http://dx.doi.org/10.1016/j.compstruc.2013.11.012

[52] Teng J.G. \& Hong T. (2006). "Postbuckling analysis of elastic shells of revolution considering mode switching and interaction." International Journal of Solids and Structures, 43, 551-568.

[53] Riks E. (1979). "An incremental approach to the solution of snapping and buckling problems" Int. J. Solids. Struct., 15, 529-551.

[54] Fujii F., Noguchi H. \& Ramm E. (2000). "Static path jumping to attain postbuckling equilibria of a compressed circular cylinder." Computational Mechanics, 26, 259-266.

[55] Rotter J.M. \& Teng J.G. (1989). "Elastic stability of cylindrical shells with weld depressions." ASCE Journal of Engineering Mechanics, 115(5), 1244-1263.

[56] Rotter J.M. \& Zhang Q. (1990). "Elastic buckling of imperfect cylinders containing granular solids." ASCE Journal of Structural Engineering, 116(8), 22532271.

[57] Knödel P., Ummenhofer T. \& Schulz U. (1995). "On the modelling of different types of imperfections in silo shells." Thin-Walled Structures, 23, 283-293.

[58] Berry P.A., Rotter J.M. \& Bridge R.Q. (2000). "Compression tests on cylinders with axisymmetric weld depressions." ASCE Journal of Engineering Mechanics, 126(4), 405-413.

[59] Holst J.M.F.G., Rotter J.M. \& Calladine C.R. (2000). "Imperfections and buckling in cylindrical shells with consistent residual stresses." Journal of Constructional Steel Research, 54, 265-282.

[60] Sadowski A.J. \& Rotter J.M. (2011). "Steel silos with different aspect ratios: I Behaviour under concentric discharge." Journal of Constructional Steel Research, 67, 1537-1544. 
Published in: Computers and Structures, 133C, 90-102.

DOI: http://dx.doi.org/10.1016/j.compstruc.2013.11.012

[61] Song C.Y., Teng J.G. \& Rotter J.M. (2004). "Imperfection sensitivity of thin elastic cylindrical shells subject to partial axial compression." International Journal of Solids and Structures, 41, 7155-7180.

[62] Rotter J.M., Cai M. \& Holst J.M.F.G. (2011). "Buckling of thin cylindrical shells under locally elevated compressive stresses." ASME Journal of Pressure Vessel Technology, 133(1), 011204-1 to 11.

[63] Pacoste C. \& Eriksson A. (1995). "Element behaviour in post-critical frame analysis." Computer Methods in Applied Mechanics and Engineering, 125, 319-343.

[64] Eriksson A. (1992). "On accurate descriptions for primary and secondary paths in equilibrium problems." Computers and Structures, 44(1/2), 229-242.

[65] Eriksson A. (1994). "Fold lines for sensitivity analyses in structural instability." Computer Methods in Applied Mechanics and Engineering, 114, 77-101.

[66] Eriksson A. (1998). "Structural instability analyses based on generalised pathfollowing." Computer Methods in Applied Mechanics and Engineerging, 156, 45-74.

[67] Eriksson A., Pacoste C. \& Zdunek A. (1999). "Numerical analysis of complex instability behaviour using incremental-iterative strategies" Computer Methods in Applied Mechanics and Engineering, 179, 265-305.

[68] Singer J., Arbocz J.\& Babcock C.D.Jr (1979). "Buckling of imperfection stiffened cylindrical shells under axial compression." AIAA Journal, 9(1), 68-75.

[69] Arbocz J. \& Babcock C.D.Jr. (1969). "The effect of general imperfections on the buckling of cylindrical shells." ASME Journal of Applied Mechanics, 36, 28-38.

[70] Arbocz J. \& Williams J.G. (1977). "Imperfection surveys on a 10-ft diameter shell structure." AIAA Journal, 15(7), 949-956. 
Published in: Computers and Structures, 133C, 90-102.

DOI: http://dx.doi.org/10.1016/j.compstruc.2013.11.012

[71] Teng J.G., Lin X., Rotter J.M. \& Ding X.L. (2005). "Analysis of geometric imperfections in full-scale welded silos." Engineering Structures, 27, 938-950.

[72] Ding. X., Coleman R. \& Rotter J.M. (1996). "Technique for precise measurement of large-scale silos and tanks." ASCE Journal of Surveying Engineering, 122(1), 14-25. 
Published in: Computers and Structures, 133C, 90-102.

DOI: http://dx.doi.org/10.1016/j.compstruc.2013.11.012

\section{FIGURE CAPTIONS}

Fig. 1 - Generic illustration of key features of the spiral welding process

Fig. 2 - Illustration of helical partitioning and meshing in ABAQUS CAE, after [29]

Fig. 3 - System geometry for a non-conformal transformation for a 'single-helix' mesh

Fig. 4 - System geometry for a conformal transformation for a 'double-helix' mesh

Fig. 5 - Meshing of the rectangular strip in the image plane

Fig. 6 - Cylindrical boundary details for the two helical mesh types (helical angle $\alpha=$ $\left.20^{\circ}\right)$

Fig. 7 - Modelling of eigenmode imperfections with regular and helical meshes (helical angle $\alpha=30^{\circ}$ )

Fig. 8 - Linear bifurcation modes for a cylinder under axial compression meshed with different helical meshes (all critical loads within $1 \%$ of the classical solution $N_{c l}$ )

Fig. 9 - Shape of mesh perturbation in the form of a linear bifurcation eigenmode, with assumed amplitude $\delta / t=0.01$ (computed using a 'rectangular' mesh)

Fig. 10 - Nonlinear equilibrium paths for an axially-compressed cylinder with 'singlehelix' meshes of varying helical angles

Fig. 11 - Nonlinear equilibrium paths for an axially-compressed cylinder with 'doublehelix' meshes of varying helical angles

Fig. 12 - Selected post-buckling deformed modes for the axially-compressed cylinder

Fig. 13 - Modelling of spiral weld imperfections in cylindrical tubes 
Published in: Computers and Structures, 133C, 90-102.

DOI: http://dx.doi.org/10.1016/j.compstruc.2013.11.012

Fig. 14 - Variation of buckling strength with helical weld depression amplitude

Fig. 15 - Incremental buckling mode for an imperfect cylinder with spiral welds at a helical angle of $\alpha=15^{\circ}$ ('double-helix' mesh). The top of each figure is at the loaded boundary of the cylinder.

Fig. 16 - Incremental buckling mode for an imperfect cylinder with spiral welds at a helical angle of $\alpha=45^{\circ}$ ('double-helix' mesh). The top of each figure is at the loaded boundary of the cylinder.

\section{TABLE CAPTIONS}

Table 1 - Nonlinear buckling loads for the axially-compressed cylinder 